\title{
British Society of Breast Radiology Annual Scientific Meeting 2013
}

\author{
Liverpool, UK. 10-12 November 2013
}

Published: 8 November 2013

These abstracts are available online at http://breast-cancer-research.com/supplements/15/S1

\section{ORAL PRESENTATIONS}

01

4.1: Nonbiopsy of presumed fibroadenomas in patients $<30$ years: is it safe? A single unit experience and review of European practice K Taylor*, H Vandersluis, P Britton, M Wallis

Cambridge Breast Unit, Cambridge University Hospitals NHS Foundation

Trust, Cambridge, UK

Breast Cancer Research 2013, 15(Suppl 1):01

Introduction: Previous research in this centre enabled the introduction of a local protocol of nonbiopsy and discharge of women $<30$ years old with presumed fibroadenomas (FAs). Four years on, we have audited protocol accuracy, effect on biopsy workload, safety and its potential for influencing UK and European practice.

Methods: Women aged $<30$ attending the breast unit between 1 February 2009 and 31 January 2013 were retrospectively identified. Clinical and imaging results were scored following national guidelines locally adapted to incorporate a nonbiopsy protocol such that presumed FAs scored E2/U2 meeting specific protocol criteria were discharged without core biopsy. In addition, a survey assessing European practice was completed by 31 centres. Results: A total of 1,571 women age $<30$ were referred to the breast unit. Seven cancers were diagnosed, all aged 25 to 29 . In total, 266 presumed FAs E2/U2, meeting nonbiopsy criteria were discharged without biopsy, 84 were aged 25 to 29. Fourteen re-attended with increase in size, none biopsied but five excised due to patient choice. Sixty-six E2/U2, probable FAs were biopsied due to noncompliance with the protocol. Of these, 54 were FAs, seven phyllodes tumours, and one cancer. The protocol resulted in a $78 \%$ reduction in biopsy workload in women aged $\geq 20$ and a $72 \%$ reduction in women aged 25 to 29 . No cancers developed in discharged patients, mean follow up 2.4 years. European survey results demonstrate $29 \%$ of respondents routinely sample FAs in women age $\geq 20,55 \%$ in women age $\geq 25$. Seventy-nine per cent of non-UK respondents follow-up FAs regardless of patient age.

Conclusion: With rigorous adherence, our nonbiopsy protocol for presumed FAs in women age $<30$ appears safe and reduces biopsy/followup workload.

\section{2}

4.2: Vacuum biopsy in the management of lobular in situ neoplasia: a single-centre experience

C Parkin ${ }^{1 *}$, S Garewal ${ }^{2}$, P Waugh ${ }^{2}$, AJ Maxwell ${ }^{2,3}$

${ }^{1}$ North Western Deanery School of Radiology, Manchester, UK; ${ }^{2}$ Royal Bolton Hospital, Bolton, UK; ${ }^{3}$ University Hospital of South Manchester, Manchester, UK Breast Cancer Research 2013, 15(Suppl 1):O2

Introduction: Lobular in situ neoplasia (LISN) is encountered with increasing frequency in core needle biopsies (CNB) of the breast. It is a generalised risk factor and probable nonobligate precursor for some breast cancers. Historically, open biopsy was performed to exclude associated malignancy. Controversy currently surrounds the management of LISN, and practice consequently varies between departments. This study is a review of a single centre's 13-year experience of managing LISN with vacuum-assisted biopsy (VAB) in order to assess the safety of this policy.

Methods: A retrospective review of the breast screening database, pathology database, high-risk patient database and patients recruited to the Sloane Project was completed. Patients with LISN as the most pertinent diagnosis on $\mathrm{VAB}$, with or without preceding 14-gauge CNB, were identified. Those with pathological results not concordant with imaging were excluded. The outcome of subsequent annual surveillance mammograms was recorded.

Results: Between February 1998 and March 2012, 42 patients had LISN as the most pertinent diagnosis at $\mathrm{VAB}$, with or without preceding CNB. No open biopsies were performed in this group. Mean radiological follow up was 39 months (range 0 to 105 months). There were no new diagnoses of breast cancer during follow up. Three patients died: one with a previous history of invasive breast cancer died from metastatic breast cancer and two died from unrelated causes.

Conclusion: In the presence of adequate tissue sampling and radiologicalpathological concordance, VAB is a safe alternative to open biopsy in the management of LISN.

03

4.3: Upgrades of B5a (non-invasive) core biopsies to invasive disease at final surgery: a retrospective review across the Scottish Breast Screening Programme

YT Sim ${ }^{1 *}$, JC Litherland

the QA Radiology Leads, Scottish Breast Screening Programme ${ }^{2}$

${ }^{1}$ West of Scotland Breast Screening Centre, Glasgow, UK; ${ }^{2}$ Scottish Breast Screening Programme, UK

Breast Cancer Research 2013, 15(Suppl 1):O3

Introduction: Women with B5a (non-invasive) preoperative core biopsies upgraded to invasive disease at surgery have a high chance of needing further surgery. The average B5a upgrade rate across UK breast screening programmes is around $20 \%$. Through this Scottish review, we aim to identify factors affecting upgrade rates and ways to improve our performance.

Methods: This was a retrospective analysis of 1,252 cases of B5a biopsies from the Scottish Breast Screening Programme between 2004 and 2012. Final surgical pathology was correlated with radiological and biopsy factors. Results: B5a upgrade rates for the units ranged from 19.2 to $29.2 \%$, with average of $23.6 \%$. Mean sizes of invasive tumours were small (3 to $11 \mathrm{~mm}$ ). Upgrade rate was significantly higher for cases where the main mammographic abnormality was mass, distortion or asymmetry, compared with microcalcification alone (33.2\% vs. $21.7 \%)(P=0.0004)$. The upgrade rate was significantly lower with use of large-volume vacuum-assisted biopsy (VAB) devices than 14 -gauge core needles $(19.9 \%$ vs. $26 \%)(P=0.013)$. The upgrade 
rate was lower in stereotactic than ultrasound-guided biopsies $(21.2 \%$ vs. $36.1 \%)(P<0.001)$.

Heterogeneity of data from different units limited evaluation of other potential factors.

Conclusion: There is variation in practice across Scottish units, including first-line biopsy technique and/or device and protocols for repeat biopsy. Upgrade rates are lower for cases with microcalcification as the sole mammographic feature, and with use of VAB devices. Nevertheless, it is of interest that a few centres maintain low upgrade rates despite not routinely using $V A B$ as the first-line technique for biopsy of microcalcification.

05

4.5: Diagnosing breast cancer in a high-risk cohort

EAM O'Flynn ${ }^{1 *}, V_{\text {Houle }}^{2}$, ARM Wilson ${ }^{2}$, AZ Evans ${ }^{3}$, SD Allen ${ }^{2}$

${ }^{1}$ Institute of Cancer Research and Royal Marsden Hospital, Sutton, UK:

${ }^{2}$ Royal Marsden Hospital, London, UK; ${ }^{3}$ Ninewells Hospital and Medical

School, Dundee, UK

Breast Cancer Research 2013, 15(Suppl 1):05

Introduction: MRI is a common method for detecting breast cancer in women at high risk $[1,2]$ These women may instead be diagnosed mammographically or present symptomatically. The aim of this study was to investigate how breast cancer is diagnosed in high-risk women and determine whether there are specific characteristics related to the type of presentation.

Methods: A total of 125 high-risk patients with 134 breast cancers (69 BRCA, 65 family history) were managed at the Royal Marsden Hospital from 1994 to 2013. Following ethical approval, data were collected retrospectively for each presentation of breast cancer: method of presentation/diagnosis (MRI, mammography, symptomatic), age at diagnosis, cancer type, grade, size, presence of DCIS, lymphovascular invasion (LVI), nodal status and tumour subtype. Chi-squared and ANOVA analyses determined any association between the parameters, $P<0.05$ was significant.

Results: Ten breast cancers were MRI detected, 43 mammography detected and 81 symptomatic (mean age 41,51 , and 45 years $(P=0.008)$; mean size 17,29 , and $34 \mathrm{~mm}(P=0.076)$ respectively).The majority of cancers were high-grade (68\%) invasive ductal carcinomas (78\%) without LVI (76\%). MRIdetected cancers were triple negative in $60 \%(P=0.03)$, node negative in $100 \%(P=0.005)$ with DCIS in $70 \%(P=0.007)$. Mammography-detected cancers were luminal in 77\% $(P=0.03)$, node negative in $77 \%(P=0.005)$, with DCIS in $81 \%(P=0.007)$. Symptomatic cancers were luminal in $54 \%$, triple negative in $41 \%$, node negative in $56 \%$ and DCIS positive in $51 \%$.

Conclusion: In this high-risk cohort, MRI detects small, triple-negative, node-negative cancers in younger women, while mammography detects larger, luminal, cancers in older women that may be node positive.

References

1. Leach MO, et al: Lancet 2005.

2. Kuhl CK, et al: JCO 2005.

\section{6}

4.6: Anisotropy in solid breast lesions at shear wave elastography: relationship to the radial plane and implications for benign/malignant differentiation

K Skerl", S Vinnicombe, P Whelehan, K Thompson, D McLean, A Evans

University of Dundee, UK

Breast Cancer Research 2013, 15(Suppl 1):06

Introduction: Anisotropy is the directional dependence of the measurement of a property. As breast tissue structure and some breast diseases (DCIS) are anisotropic in structure, we aimed to establish the frequency, degree and diagnostic value of shear wave elastography anisotropy in solid breast lesions.

Methods: Ninety-eight solid breast lesions ( 31 benign and 67 malignant) were examined in the radial and anti-radial planes, with two mean stiffness measurements (in $\mathrm{kPa}$ ) being taken in each plane and averaged. The difference between the radial and anti-radial measurements was squared to make all readings positive, and compared with the histological diagnosis. Paired Student $t$ tests and chi-square tests were performed to establish statistical significance of the relationships.
Results: Anisotropy was found in both benign and malignant lesions. However, the stiffness values were not related to the examination plane in either group of lesions $(P=0.2)$. Anisotropy was greater in malignant lesions than benign lesions $(P<0.0001)$. Using a malignancy threshold value for the square of the difference in radial and anti-radial stiffness of 200, the sensitivity, specificity and diagnostic accuracy of the presence of this level of anisotropy were $72 \%, 87 \%$ and $77 \%$ respectively.

Conclusion: Stiffness of solid breast lesions on shear wave elastography is not directly related to the examination plane. Malignant lesions show more anisotropy than benign lesions. Therefore, adding anisotropy to other shear wave parameters has the potential to improve the ability of this modality to differentiate benign from malignant solid breast lesions.

\section{POSTER PRESENTATIONS}

P1

PB.01: Second-look ultrasound examination of the breast following MRI: MR and sonographic findings

K Alrawi i, J Bansal, P Young

Cardiff \& Vale Breast Centre, University Hospital Llandough, Cardiff \& Vale University Health Board, Cardiff, UK

Breast Cancer Research 2013, 15(Suppl 1):P1

Introduction: MRI is widely accepted to be the most sensitive imaging modality for detecting breast cancer, but has relatively low specificity. Often additional enhancing areas are identified on MRI that require further investigation. In this study we evaluated second-look ultrasound following breast MRI and the impact of this on patient management.

Methods: A retrospective review was undertaken of all breast MRIs performed between July 2010 and December 2012. Patients who had further evaluation with second-look ultrasound were reviewed. Clinic letters were also reviewed to identify any subsequent change in patient management

Results: A total of 261 breast MRI scans were performed over this time period. Fifty-two (19.9\%) had a second-look ultrasound performed; 24 for mass-like lesions and 28 for nonmass-like lesions identified on MRI. In total, $18 / 24$ (75\%) mass-like lesions had a corresponding ultrasound abnormality, and $12 / 28(42.8 \%)$ nonmass-like lesions had a corresponding ultrasound abnormality. Thirty biopsies were performed (57.6\%) and of these nine (17.3\%) were malignant. Malignant lesions were equally distributed between mass-like and nonmass-like lesions. In total, 7/9 malignant lesions were scored U4 or U5, demonstrating features suspicious of malignancy. Management was altered in all nine cases.

Conclusion: MR-directed second-look ultrasound is a valuable tool in diagnostic work-up. Mass-like lesions on MR are more likely to have an ultrasound correlate. Most malignant lesions had clearly malignant features on ultrasound.

\section{P2}

PB.02: Magnetic resonance imaging axilla: friend or foe?

CF Hathaway ${ }^{1 *}$, C Zammit ${ }^{2}$, S Shaheed ${ }^{2}$, G Rubin $^{3}$, J O'Brien

${ }^{1}$ Brighton and Sussex Medical School, Brighton, UK; ${ }^{2}$ Department of Surgery,

Park Centre for Breast Care, Brighton, UK; ${ }^{3}$ Department of Radiology, Park

Centre for Breast Care, Brighton, UK

Breast Cancer Research 2013, 15(Suppl 1):P2

Introduction: Breast MRI is used for local staging of breast cancer. It also gives information on the axilla. We looked at patients whose staging breast MRI revealed indeterminate axillary node appearances.

Methods: A retrospective analysis was conducted of all breast MRIs in one institution between July 2012 and June 2013. MRI scans that found indeterminate axillary nodes and recommended second-look ultrasound were analysed, and correlated with fine-needle aspiration (FNA) cytology and surgical histology results.

Results: A total of $180 \mathrm{MRI}$ scans were performed for local breast cancer staging, having met NICE early cancer guidelines. Ten had incomplete follow up, leaving 170 in the study. In total, 131 correlated with the primary axillary ultrasound imaging and cytology, and did not have further axillary investigation. Thirty-nine (22.9\%) requested second-look ultrasounds, with 25 requiring FNA; 10 (5.9\%) found positive, 15 (8.8\%) 
found negative nodes. Nodes found to be positive on FNA had axillary clearance rather than sentinel node biopsy, confirmed on surgical histology in all 10. Of the 14 patients who did not have an FNA on second look, only one was found to be positive on later histology. Two of the 15 had FNA on second look, and given a negative result, did ultimately have positive nodes on sentinel node biopsy.

Conclusion: MRI and second-look ultrasound/FNA correctly identified $10(5.9 \%)$ more patients with involved nodes than initial staging. Twentynine $(17.1 \%)$ other patients had additional axillary ultrasound including FNA in $15(8.8 \%)$ without a change in axillary management.

\section{P3}

PB.03: Evaluating the role of breast MRI at mid-cycle chemotherapy D Santosh ${ }^{1 *}$, G Uruski $^{1}$, A Borley ${ }^{1,2}$, P Young ${ }^{1}$

${ }^{1}$ Cardiff and Vale University Health Board, Cardiff, UK; ${ }^{2}$ Velindre NHS Trust, Cardiff, UK

Breast Cancer Research 2013, 15(Suppl 1):P3

Introduction: The aim was to evaluate whether breast magnetic resonance imaging (MRI) following mid-cycle chemotherapy was influencing the neoadjuvant chemotherapy (NAC) regimen.

Method: Thirty-two patients with breast malignancy receiving NAC were included in this retrospective study. Baseline and mid-cycle MRI were performed. NAC regimen and breast MRI reports were obtained from an online database. Tumour response was assessed by calculating percentage of volume reduction between interval scans. The response was categorised arbitrarily as good, moderate and poor with $>50 \%, 20$ to $50 \%$ and $<20 \%$ reduction in tumour volumes respectively.

Results: Nine patients had second tumours detected on the baseline MRI. The index tumours $(n=32)$ had showed good response in $23(71.9 \%)$, moderate response in four (12.5\%) and poor response in five (15.6\%) on their mid-chemotherapy MRI. The five poor responders had good response in either the axillary node or in the second tumour. The second tumours $(n=9)$ showed good response in six $(66.7 \%)$, moderate in two $(22.2 \%)$ and poor in one (11.1\%). No changes were made to chemotherapy regimen based on the MRI findings and these percentage tumour volume reductions.

Conclusion: Our study shows that the percentage reduction in the tumour volumes demonstrated by the interval MRI scans are an important determinant of response to NAC. It is possible to obtain similar information from newer $4 \mathrm{D}$ sonography, which is cheaper and quicker to perform than $\mathrm{MRI}$, and is better tolerated. In our practice, assessment at the midchemotherapy point is now undertaken with clinical assessment and ultrasound, which have provided adequate information regarding tumour responses to chemotherapy.

\section{P4}

PB.04: MRI In lobular and mixed lobular/ductal carcinomas: can we preselect cases based on imaging appearance?

MM Hoosein*, L Grosvenor, D Lister, M Al-Attar

University Hospitals of Leicester, UK

Breast Cancer Research 2013, 15(Suppl 1):P4

Introduction: Lobular carcinoma presents a diagnostic challenge. Imaging and clinical findings are usually subtle. In the current climate it imposes extra strain on our resources. Our aim was to assess contribution of MRI in the preoperative local staging of lobular and mixed lobular/ductal carcinoma and to evaluate whether we can select cases for preoperative MRI based on mammographic appearance.

Methods: A retrospective review of data provided by the local breast cancer database was performed. Patients with confirmed lobular or mixed lobular/ductal carcinomas that had MRI staging during a 5-year period were identified. Imaging and histopathology reports were reviewed. A total of 381 cancers were diagnosed in the study period. Ninety-one patients had breast MRI. Four cases were excluded as no final histology was available. Eightyseven patients (mean age 58) with 89 involved breasts constituted our study population.

Results: Breakdown of mammographic lesions was as follows: PD, 39 (43.8\%); masses, 20 (22.4\%); ASD, 18 (20.2\%); lymphoedema, one (1.1\%); calcifications, two (2.2\%); occult/subtle, nine (10.1\%). MRI had a positive contribution in $27 / 83$ cases (32.5\%), did not add any further information in $49 / 83$ cases (59\%) and a negative contribution in only $7 / 83$ cases (8.4\%). MRI notably identified greater disease extent, multifocal and contralateral disease. MRI was most useful in assessing disease extent when lobular carcinoma presented as PD or mammographically occult and was of least benefit when presenting as a mass lesion.

Conclusion: MRI had no advantage over conventional imaging in the majority of lobular and mixed lobular/ductal cancers presenting as a focal mass lesion. Its application could be tailored more specifically to assess nonmass lesions.

\section{P5}

PB.05: MRI-guided vacuum-assisted breast biopsy at 3T: initial experience

M Sreenivas

University Hospitals Coventry and Warwickshire NHS Trust, Coventry, UK Breast Cancer Research 2013, 15(Suppl 1):P5

Introduction: As the specificity is relatively low, histological confirmation of incidental breast lesions at $3 \mathrm{~T}$ is needed if the treatment plan has to change. MRI-guided biopsy is needed as a proportion of these lesions will not been seen on conventional imaging.

Methods: Forty-nine out of 240 patients undergoing DCE-MRI studies performed between 1 July 2011 and 20 July 2013 warranted second-look US ( $20 \%$ of patients). Fifteen of the 49 patients underwent MRI-guided biopsy predominantly as second-look US was negative. Diagnostic imaging and biopsy were performed on GE 3 T using a dedicated breast coil, grid method, CADstream software and Vacora vacuum-assisted biopsy device with a $10 \mathrm{G}$ needle obtaining between 10 and 22 cores.

Results: Fourteen of the 15 patients had technical success (in one patient the biopsy had to be performed twice due to unsatisfactory sampling, and repeat biopsy yielded $\mathrm{B} 5 \mathrm{~b}$ ).

Seven nonmass $\mathrm{M} 3$ lesions yielded a $43 \%$ malignancy rate $(B 4=1$ and $B 5 a=2)$. Two of five $M 4$ lesions were masses yielding $B 5 a$ with overall malignancy yield in this category of $40 \%$. One of the two M5 masses was B5b with a cancer yield in this category of $50 \%$. Overall malignancy yield was $43 \%$.

Conclusion: As far as we know we are the only unit in the UK to perform MRI-guided breast biopsy at $3 \mathrm{~T}$ and our results are in accordance with published literature (cancer yield between 24 and $40 \%$ ). This preliminary work has shown that MRI biopsy at $3 \mathrm{~T}$ is feasible using a hand-held multiple-insertion vacuum-assisted device.

P6

PB.06: Preliminary results in the performance comparison between the size ratio and colour scoring breast ultrasound elastographic techniques

LCH Leong", THL Moey, LSJ Sim

Singapore General Hospital, Singapore

Breast Cancer Research 2013, 15(Suppl 1):P6

Introduction: There are a few breast ultrasound elastographic techniques that can help with distinguishing malignant breast lesions from benign ones. Two of the better known ones are the size ratio and colour scoring methods. The aim of this study is to prospectively compare the diagnostic performance of these two elastographic techniques.

Methods: Female patients referred to the radiology department for imageguided breast biopsy were prospectively evaluated with ultrasound elastography prior to biopsy following informed consent. The two elastographic methods were assessed on each breast lesion separately by different radiologists. A size ratio of $\geq 1.1$ was taken to be malignant and $<1.1$ was benign. Colour scores of 1 to 3 were taken as benign and colour scores of 4 to 5 were considered malignant. Histological diagnosis was used as the gold standard. The sensitivity and specificity of both techniques were compared using the Fisher's exact test.

Results: Sixty-five breast lesions in 63 women were evaluated at the interim stage of the study. There were 21 malignant and 44 benign breast lesions. The sensitivity and specificity of the size ratio technique were $100 \%(21 / 21)$ and $81.8 \%(36 / 44)$. The sensitivity and specificity of the colour scoring method were $57.1 \%(12 / 21, P=0.001)$ and $86.4 \%(38 / 44, P=0.772)$. 
Conclusion: The preliminary results indicate that the size ratio elastographic technique is more sensitive and accurate than the colour scoring method. A larger study is ongoing.

\section{P7}

PB.07: Why are some fibroadenomas stiff using shear wave elastography?

M Elseedawy ${ }^{3^{*}}$, P Whelehan ${ }^{1,2,3}$, S Vinnicombe ${ }^{1,2,3}$, K Thomson $^{1,2,3}$, A Evans ${ }^{1,2,3}$ 'Dundee Cancer Centre, Dundee, UK; ${ }^{2}$ Ninewells Hospital, Dundee, UK;

${ }^{3}$ University of Dundee, UK

Breast Cancer Research 2013, 15(Suppl 1):P7

Introduction: Shear wave elastography (SWE) is a promising modality for differentiating benign from malignant breast masses. A proportion of fibroadenomas are stiff (mean stiffness $>50 \mathrm{kPa}$ ), resulting in false positive SWE findings. The aim of this study was to identify which features of fibroadenomas are associated with false positive SWE findings.

Methods: A total of 151 patients with histologically confirmed fibroadenomata were identified from a prospective database, from a single breast unit. The following features were assessed by a single observer who was unaware of the SWE findings: Patient age, greyscale ultrasound lesion diameter $(<15 \mathrm{~mm}$ or $\geq 15 \mathrm{~mm})$, distance from the lesion to skin composition of surrounding tissue (fatty, mixed or dense) and source of referral (screening or symptomatic). Statistical analysis was carried out using the chi-square test.

Results: A statistically significant association was found between greyscale ultrasound lesion size and lesional stiffness. Twenty-nine of $70(41 \%)$ lesions $\geq 15 \mathrm{~mm}$ were stiff, versus 10 of $81(12 \%)<15 \mathrm{~mm}, P=0.001$. Patient age, distance from the lesion to skin, makeup of surrounding tissue and source were not significantly associated with stiffness.

Conclusion: Fibroadenomas giving false positive SWE results tend to be larger in size than these that do not. More compression of adjacent normal tissue is assumed to be the cause of our findings. As previous studies have shown that large cancers tend to be stiffer than smaller cancers, it may be appropriate to vary the quantitative cutoff value used for benign/malignant differentiation in SWE according to lesion size.

P9

PB.09: Clinical audit of technical recall data for blur following the introduction of the breath hold technique in breast screening

LE Bisset

Dorset Breast Screening Unit, Poole, UK

Breast Cancer Research 2013, 15(Suppl 1):P9

Introduction: Digital mammography has increased the number of technical recall (TR) appointments due to blurred images. Within a regional breast screening unit, $177 / 29,314$ women $(0.6 \%)$ were recalled in an analogue 12-month period compared with 639/30,102 (2.12\%) with digital. Current NHSBSP TR standard $=3 \%$, target $=2 \%$ [1]. A retrospective audit to assess breath-hold technique TR data aimed to: measure and record data for the TR's pre and post breath hold; compare these results with NHSBSP standards; and make recommendations for future practice based on these results.

Methods: Datasets gathered information that included mammographic view, radiographer, side of blur, compressed breast thickness, force and location. The data for the pre breath hold sample $(8,467)$ and post breath hold sample $(9,072)$ were compared. A retrospective questionnaire of mammographers' perceptions demonstrated the technique was easy and rarely added additional time.

Results: Pre breath hold there were 104/8,467 recalls for blurring, and post breath hold there were $31 / 9,072$. The results demonstrate recalls measured against the NHSBSP targets, and TRs dropped from $3.21 \%$ (above recommended practice) to $2.08 \%$ (in line with standard/almost target recommendations). This is a $69.1 \%$ reduction. Fisher's exact test and Pearson's chi-squared with Yates' continuity both produced $P<2.2 \times 10^{-16}$. Both were therefore statistically significant for blur.

Conclusion: The breath hold technique has reduced the number of TRs for blur. Therefore, it is recommended that this technique should be adopted across the entire NHSBSP.
Reference

1. NHSBSP: Consolidated Guidance on Standards for the NHSBSP Sheffield: NHS Cancer Screening Programmes 2005, Publication Number 60.

P10

PB.10: Breast compression, compressed breast thickness and volumetric breast density

J Khan-Perez ${ }^{1 *}$, C Mercer $^{2}$, M Bydder $^{2}$, J Sergeant ${ }^{3}$, J Morris ${ }^{4}$, A Maxwell $^{2}$,

C Rylance ${ }^{2}$, S Astley ${ }^{3}$

${ }^{1}$ The University of Manchester Medical School, Manchester, UK; ${ }^{2}$ Nightingale

Breast Centre and Genesis Prevention Centre, UHSM, Manchester, UK;

${ }^{3}$ Centre for Imaging Sciences, Institute of Population Health, University of

Manchester, UK; ${ }^{4}$ Department of Medical Statistics, University Hospital of

South Manchester, Wythenshawe Hospital, Manchester, UK

Breast Cancer Research 2013, 15(Suppl 1):P10

Introduction: Adequate compression of the breast during mammography is essential both for ensuring high-quality images and for accurate assessment of breast density using automated volumetric breast density software. Here we explore the relationship between breast density and imaging parameters.

Method: We identified a set of 210 women undergoing routine screening mammography by the same experienced radiographer using the same mammography unit. Breast density data (breast volume, fibroglandular tissue volume) were obtained using Volpara ${ }^{\mathrm{TM}} 1.4 .0$ and imaging parameters (compression force, breast thickness and dose) were extracted from the DICOM headers. Statistical analysis using Spearman's rank-order coefficient was used to examine any existing relationships.

Results: There were significant positive correlations $(P<0.01)$ between both breast volume and gland volume with compressed breast thickness, $X$-ray dose and compression force $(P<0.05)$. Volumetric breast density was negatively correlated with compression force in the CC view $(P<0.01)$ and thickness in all views $(P<0.01)$, and breast volume had a significant positive correlation with gland volume $(P<0.01)$.

Conclusion: Our results show that large, dense breasts had greate thicknesses, higher X-ray doses and required a greater compression force during mammography. There was insufficient evidence to determine whether higher compression forces in larger breasts were due to increased glandular content. The negative correlations with volumetric breast density expressed as the percentage of the breast volume occupied by dense fibroglandular tissue can be explained by the fact that the positive correlations with breast volume were stronger than those with gland volume.

P11

PB.11: Identifying women at high risk of developing breast cancer: implications of adjusting for inter-observer variability in visual analogue scale assessment of percentage breast density JC Sergeant ${ }^{1 *}$, M Sperrin ${ }^{2}$, L Bardwell ${ }^{3}$, I Buchan ${ }^{2}$, DG Evans ${ }^{4}$, A Howell $^{4}$ SM Astley ${ }^{1}$

${ }^{1}$ Centre for Imaging Sciences, Institute of Population Health, University of Manchester, UK; ${ }^{2}$ Centre for Health Informatics, Institute of Population Health, University of Manchester, UK; ${ }^{3}$ Department of Mathematics and Statistics, University of Lancaster, UK; ${ }^{4}$ Nightingale Centre and Genesis Prevention Centre, University Hospital of South Manchester, UK Breast Cancer Research 2013, 15(Suppl 1):P11

Introduction: Breast density is a well-established risk factor for breast cancer, with assessment of percentage density via a visual analogue scale (VAS) a practical method of measurement strongly associated with risk. We present a method to adjust for inter-observer differences in VAS density estimates and examine the effect of adjustment on the classification of women at high risk of developing breast cancer.

Methods: A two-stage method is used to make estimates by different observers comparable. Results from all observers are transformed onto the same distribution, then differences in case mix are accounted for. We applied our approach to 13 experienced readers assessing 13,694 screening mammograms from a large clinical study where women are categorised as high risk if they have a 5 to $8 \% 10$-year risk computed by a validated risk model and their breast density is in the top decile of the study population. 
Results: A total of 1,125 women were assessed as having a 10-year risk of 5 to $8 \%$. Initially 126 of these were also high density, therefore classified as high risk, rising to 147 after density adjustment. After adjustment, 35 women were reclassified from nonhigh to high risk (3.5\% of those initially nonhigh risk) and 14 women were reclassified from high to nonhigh risk ( $11.1 \%$ of those initially high risk).

Conclusion: Adjusting VAS estimates of breast density for inter-observer variation substantially affected which women were classified as high risk of developing breast cancer. If VAS assessment of density is to be used in risk assessment to inform screening strategies, adjustment must be considered.

\section{P13}

PB.13: Comparison between analogue and digital mammography: a reader's perspective

H Gay", R Pietrosanu, S George, D Tzias, R Mehta, C Patel, S Heller, L Wilkinson St George's Hospital NHS Trust, London, UK

Breast Cancer Research 2013, 15(Suppl 1):P13

Introduction: In comparison with analogue film, digital mammography provides images with more contrast and allows image manipulation. This study compares features identified on digital and analogue mammograms. Methods: During the transition to digital mammography between June and August 2011, women who had received analogue screening mammography and required further views at assessment had repeat digital images. Eight readers reviewed digital and analogue images separately, marking all features that caught their attention on a pro forma. Readers commented on breast density and characterised all features using BiRads descriptors.

Results: Twenty-three women were recalled for soft tissue lesions (six malignancies) and 13 for calcifications (one malignancy). Fifty analogue/ digital single-view mammogram pairs were obtained, 11 included histologically malignant abnormalities. Breast density scores were lower on digital than analogue $(P<0.001)$. There was no significant difference in the descriptions of calcifications. More inconsequential soft tissue features were described on analogue. Soft tissue features tended to be scored as less conspicuous on analogue than digital images. There was no significant difference in the description of 5/6 soft tissue cancers, but one cancer was seen by five readers on analogue, and only two on digital mammogram. See Table 1.

Conclusion: This study showed that readers reported breasts as less dense and identified fewer distracting soft tissue lesions on digital mammography but there was no difference in the reporting of calcifications. One of six cancers was under-reported on digital mammography.

\section{P14}

PB.14: Visibility of screen-detected invasive carcinoma on digital breast tomosynthesis: do we need two views?

O Abeyakoon", D Evans, R Rahim, J Morel, R Wasan, A lqbal, J Golligher,

C Peacock, M Michell

Kings College Hospital, London, UK

Breast Cancer Research 2013, 15(Suppl 1):P14

Introduction: Digital breast tomosynthesis (DBT) increases the sensitivity and specificity of detecting invasive breast carcinoma. Integration into screening raises questions. Should we perform two-view full-field digital mammography (FFDM) and two-view DBT or two-view FFDM and singleview DBT at every screening? DBT is shown to offer greatest benefit in the assessment of a soft tissue lesion. We routinely use two-view DBT in combination mode for all patients recalled from screening for a soft tissue abnormality. The aim of our study is to assess the need for two-view DBT in the detection of breast cancer.

Table 1(abstract P13) Aggregate features identified (number of times features were marked)

\begin{tabular}{lll}
\hline & Analogue & Digital \\
\hline Soft tissue & 398 & 319 \\
Calcifications & 178 & 173 \\
\hline
\end{tabular}

Methods: We retrospectively queried our histopathology database for all screen-detected invasive cancers between 2011 and 2012. The study sample was 254 cases. Comparisons were made between the visibility of the cancer on MLO/CC DBT and histological type/grade, molecular profile and breast density (BIRADS).

Results: The mean age was 59 years. In total, $4 / 254$ cancers were visible on one-view DBT (1.6\%). (Two seen on MLO DBT [spiculated masses] and two seen only on the CC view [distortions]). A total of 11/254 had greater visibility on one view in comparison with the other view on DBT (4.3\%). Two of 254 cases were occult on FFDM and DBT. Recall was for clinical symptoms, both lobular invasive carcinoma $(0.79 \%)$. There was no relationship between histological type, grade or molecular characteristics and the visibility on one-view versus two-view DBT.

Conclusion: A total $98.4 \%$ of cancers were seen on two-view FFDM and MLO-DBT. Integration of MLO-DBT into breast cancer screening with twoview FFDM is a consideration.

P15

PB.15: Pain in mammography: where and why does it arise?

D O'Leary", Z Al Maskari

University College Dublin, Ireland

Breast Cancer Research 2013, 15(Suppl 1):P15

Background: The purpose of the study was to determine the intensity of pain experienced by women undergoing mammography examination through investigation of biological, psychological and technical factors that influence any pain felt during the examination.

Methods: Sixty-four women presenting for diagnostic and screening mammography were examined. Pain experience data were collected at three discrete time-points during mammography using both a visual analogue and Likert scales.

Results: Pain due to compression was rated by $96.6 \%$ of women. The pain/discomfort averaged between mild $(42 \%)$ to moderate $(49 \%)$ in the craniocaudal (CC) projection and between mild $(22 \%)$, moderate $(61 \%)$ to very severe pain $(13.5 \%)$ in the mediolateral oblique (MLO) projection. Correlation identified significant associated factors for pain: women's age $(P=0.001)$, menstruation $(P=0.042)$, menopausal status $(P=0.002)$ and marital status $(P=0.000)$. A unique aspect was the investigation of the sources of pain in each projection. Pain experienced during breast compression mainly arises from compression pressing on the middle of the breast and chest wall in the $\mathrm{CC}$ and from the compression pressing on the sternum, middle and underside of the breast and the axilla in the MLO.

Conclusion: This study supports other published work that women experience pain during breast compression within mammography and that the radiographer plays a major role in the women's experience of pain. The study contributes to the pain debate by showing where the pain arises in the breast specifically during compression and the degrees of pain felt.

\section{P16}

PB.16: Contrast-enhanced digital mammography: a single-centre experience

SB O'Neill", DC O'Neill, N Marshall, L Duddy, MF Ryan, JE Barry

Cork University Hospital, Cork, Ireland

Breast Cancer Research 2013, 15(Suppl 1):P16

Introduction: Contrast-enhanced mammography is a relatively novel technique. We describe a single-centre experience with use of the technique to facilitate the detection and characterisation of lesions in the breast by triangulation of contrast-enhanced mammography, biopsy/gross pathological specimen and MR findings.

Methods: Retrospective review of the pathological and imaging findings of consecutive patients undergoing contrast mammography was performed. Sixty-four contrast mammograms were performed on 61 patients (all female, mean age $54.3 \pm 13.2$ years) over a 15 -month period. Technique involves a dual energy acquisition of CC and MLO views following the administration of contrast medium, then digital subtraction (GE Senographe DS).

Results: At contrast-enhanced digital mammography, enhancement was observed in 37 of 43 patients with biopsy-proved cancers. Of the six patients 
with a cancer diagnosis but no enhancement, three were post neoadjuvant treatment. Three patients without a tissue diagnosis of malignancy demonstrated mammographic enhancement. Fourteen patients also underwent gadolinium-enhanced breast MR. Nine out of 10 patients with MR enhancement also demonstrated enhancement on contrast mammogram. One patient with no MRI enhancement had enhancement on contrast mammography. Morphology generally correlated with the pathologic diagnosis.

Conclusion: The results of this study demonstrate the utility of contrastenhanced mammography in the identification, evaluation and follow-up of breast lesions. It offers similar enhancement characteristics to MRI and represents a feasible alternative in centres without onsite MRI.

P17

PB.17: Inter-observer agreement in visual analogue scale assessment of percentage breast density

JC Sergeant ${ }^{*}$, M Wilson ${ }^{2}$, N Barr ${ }^{2}$ U Beetles ${ }^{2}$, C Boggis ${ }^{2}$, S Bundred ${ }^{2}$

M Bydder², S Gadde², E Hurley², A Jain², Y Lim², L Lord², V Reece'², DG Evans², A Howell ${ }^{2}$, SM Astley ${ }^{1}$

${ }^{1}$ Centre for Imaging Sciences, Institute of Population Health, University of

Manchester, UK; ${ }^{2}$ Nightingale Centre and Genesis Prevention Centre,

University Hospital of South Manchester, UK

Breast Cancer Research 2013, 15(Suppl 1):P17

Introduction: Breast density is an important risk factor for breast cancer. Assessment of density at screening could help identify women at increased risk of cancer, who may benefit from screening with shorter intervals or different modalities. Visual analogue scale (VAS) assessment of percentage density by observers is straightforward to implement and strongly associated with cancer risk. However, using VAS assessment for stratification would require reproducibility between observers. We examine agreement between observers assessing VAS density.

Methods: The VAS breast density of 120 screening cases with full-field digital mammograms was independently assessed by 12 experienced mammographic readers. The agreement between the readers was assessed using Bland-Altman limits of agreement and the concordance correlation coefficient (CCC). The VAS densities were also converted to BIRADS breast composition categories and agreement measured with Cohen's weighted kappa.

Results: The greatest difference between two estimates for the same case was 67.75 percentage points, while the mean difference between reader pairs ranged from 0.76 to 28.58 percentage points. The $95 \%$ limits of agreement between reader pairs were $(-6.96,18.62)$ at their narrowest and $(-59.13,1.97)$ at their widest. Pairwise CCC values ranged from 0.44 to 0.92 , while the overall CCC was 0.70 . Pairwise kappa values for the BIRADS classification ranged from 0.37 to 0.84 , with a mean of 0.66 .

Conclusion: Substantial lack of agreement was found between readers visually assessing percentage breast density. This study demonstrates the need for reader harmonisation, either by training or by adjustment of results, should VAS density assessment be used for risk stratification.

P18

PB.18: Factors affecting breast density assessment

L Beattie ${ }^{1 *}$, M Bydder ${ }^{2}$, JC Sergeant ${ }^{3}$, A Maxwell ${ }^{2}$, N Barr ${ }^{2}$, U Beetles ${ }^{2}$

CRM Boggis², S Bundred², S Gadde'2, E Hurley², A Jain², E Lord², V Reece², M Wilson', P Stavrinos', DG Evans ${ }^{2,4}$, A Howell ${ }^{2,5}$, SM Astley ${ }^{2,3}$

${ }^{1}$ University of Manchester Medical School, Manchester, UK; ${ }^{2}$ Nightingale

Centre and Genesis Prevention Centre, University Hospital of South

Manchester, UK; ${ }^{3}$ Centre for Imaging Sciences, University of Manchester, UK;

${ }^{4}$ Department of Genetics, St Mary's Hospital, Manchester, UK; ${ }^{5}$ Institute of

Cancer Sciences, University of Manchester, UK

Breast Cancer Research 2013, 15(Suppl 1):P18

Introduction: High breast density, where there is a relatively large proportion of fibroglandular tissue in the breast, is associated with increased risk of developing cancer. There are several methods of assessing breast density from mammograms, and as these sometimes disagree about whether density is high (or low), we have investigated potential causes of disagreement.
Methods: A set of 6,422 mammograms with density assessed visually by two readers using Visual Analogue Scales, and volumetric breast density measured using Quantra ${ }^{\mathrm{TM}}$ and Volpara ${ }^{\mathrm{TM}}$ was obtained from the PROCAS (Predicting Risk Of Cancer At Screening) database. Cases were ranked from the highest to lowest density by each method. For each pair of methods the 20 cases with the largest discrepancy in rank, and the 20 with the smallest, were selected. Image features were recorded and compared.

Results: The two volumetric methods were more likely to disagree when calcification was present and the inframammary fold was poorly positioned. When comparing Quantra ${ }^{\mathrm{TM}}$ to visual assessment, there were more skin folds and a higher compressed breast thickness in the discrepant cases. Comparing Volpara ${ }^{\mathrm{TM}}$ with visual assessment, there were more suboptimal inframammary folds and higher compression forces in the discrepant group.

Conclusion: Although visual and volumetric methods are unlikely to produce similar density estimates, those ranked highly by one method should correspond to the high-density cases identified by another. Our study indicates the need for further investigation, as lack of ground truth means that in cases of disagreement it is not possible to tell which method produced better density estimates.

P19

PB.19: Investigation of a novel method for breast discomfort reduction during mammography

D O'Leary*, Z Al Maskari

University College Dublin, Ireland

Breast Cancer Research 2013, 15(Suppl 1):P19

Introduction: The purpose was to determine the effectiveness of a silicon cushion in providing pain relief during mammography when used to cover sharp edges on the image receptor and/or compression paddle. The impact of the silicon pad(s) on image quality and radiation dose was assessed.

Methods: The transparent silicon pad was randomly assigned to the right/ left breast of the patient; the other breast was imaged as normal. The pad (s) was strategically placed on the mammography machine using three methods. Pain experience data were collected at three discrete time-points during mammography using a visual analogue and Likert scales. Radiologist image evaluators were blinded to pad assignment for image quality evaluation. Radiation dose to the breast was compared with the pad and without.

Results: Quantitatively, no significant reduction $(P>0.05)$ was observed in the pain experienced due to the silicon pad in either mammographic projection. Qualitatively, there was a trend for pain reduction with the silicon pad. No statistically significant degradation in image quality was assessed in either projection except due to the pad design. There were, however, significant increases in the radiation dose $(P<0.00)$ for both projections due to the slight increase in the compressed breast thickness due to pad thickness.

Conclusion: The silicon breast cushion requires significant design changes before commercial use for pain reduction intervention in mammography. The study did, however, emphasise that the radiographer plays an important role in the women's experience and communication by the radiographer helps in qualitatively reducing the women's experience of pain during the examination.

\section{P20}

PB.20: Accuracy of specimen radiograph in determining lesion presence in excised specimens, correlating histological and radiological margins

L Sundaram*, N Hartley, M Hoosein, M Al-Attar, E Denton, L Grosvenor, D Lister, G McDonald, S Kaneri

Breast Care Centre, Glenfield Hospital, UHL, Leicester, UK

Breast Cancer Research 2013, 15(Suppl 1):P20

Introduction: Radiography of the excised specimen post localisation determines whether the target lesion has been removed [1]. The surgical specimen is oriented and if a margin appears suspicious on imaging, further 
tissue is removed [2]. An incompletely excised carcinoma adds to further surgical workload, psychological and physical morbidity for patients.

Methods: All patients with biopsy-proven carcinoma undergoing therapeutic excision over a 16-month period were reviewed. A retrospective review of the mammographic lesion localised, visualisation on specimen radiograph (SR), SR report on adequacy, margins of excision, histology of tumour, margins after WLE and further surgery (including minimum distances on margins) was performed and recorded.

Results: Of 68 therapeutic wire-guided excisions, lesion was accurately detected in SR in $67 / 68$ cases (99\%), comparable with the published literature. In 53/68, no more excision was recommended on imaging. Of these, histology confirmed clear margins in $45 / 68$. Further immediate excision was correctly advised in 15 patients. New excision margins were clear in 10/15 cases, thus avoiding second surgery for this subgroup. Extensive disease was seen despite re-excision in five patients. Accuracy of commenting on tumour at margins was $60 / 68(88 \%)$.

Conclusion: Discrepancy between radiological and histological margins has significant implications. Accurate SR interpretation is vital. Meticulous attention to the protocol will reduce errors.

References

1. Quality Assurance Guidelines in Breast Cancer Screening NHSBSP Publication Number 20. Sheffield: NHS Cancer Screening Programmes, 32003.

2. White J, et al: Compliance with breast conservation standards for patients with early breast carcinoma. Cancer 2003, 97:893-904.

P21

PB.21: Relationship between volumetric breast density and socioeconomic status

L Samuels ${ }^{1 *}$, SM Astley ${ }^{1}$, A Maxwell ${ }^{2}$, JC Sergeant ${ }^{1}$, J Morris $^{3}$, M Wilson $^{2}$,

P Stavrinos ${ }^{2}$, DG Evans' ${ }^{2}$, A Howell ${ }^{2}$, M Bydder ${ }^{2}$

${ }^{1}$ University of Manchester, UK; ${ }^{2}$ Nightingale Centre and Genesis Prevention

Centre, University Hospital of South Manchester, UK; ${ }^{3}$ University Hospital of South Manchester, UK

Breast Cancer Research 2013, 15(Suppl 1):P21

Introduction: Women of higher socioeconomic status (SES) are at increased risk of developing breast cancer, but it is unclear to what extent this is mediated by differences in mammographic breast density, a wellestablished breast cancer risk factor. Here we explore the relationship between volumetric breast density and SES.

Methods: Data from 6,398 perimenopausal/postmenopausal women undergoing routine NHSBSP screening were obtained from those consenting to the PROCAS (Predicting Risk Of Cancer At Screening) study. SES was assessed by obtaining the Index of Multiple Deprivation (IMD) associated with each participant's postcode. Volumetric breast density was measured using Volpara ${ }^{T M}$. The association between volumetric breast density and IMD, adjusting for age, HRT use and BMI, was assessed using linear regression. Results: A very weak negative relationship was seen between volumetric breast density and IMD (B coefficient $-0.023, P<0.001$ ), suggesting that increasing deprivation (lower SES) is associated with reduced breast density. A decreasing trend in IMD was seen with Volpara ${ }^{\mathrm{TM}}$ VDG breast density category (Table 1). This relationship was mainly driven by a negative association between SES and BMl, although unaffected by age or current HRT use.

Conclusion: SES (as measured by IMD) is not strongly associated with breast density. Established SES gradients in breast cancer risk are likely to be related to other SES-related factors, primarily BMI, rather than mammographic breast density.

Table 1(abstract P21)

\begin{tabular}{llll}
\hline $\begin{array}{l}\text { Volpara } \\
\text { grade }^{\mathrm{Tm}} \text { density }\end{array}$ & $\begin{array}{l}\text { Volpara }^{\mathrm{Tm}} \text { breast } \\
\text { density (\%) }\end{array}$ & $\begin{array}{l}\text { Mean } \\
\text { IMD }\end{array}$ & $\begin{array}{l}\text { Number (\%) of } \\
\text { women }\end{array}$ \\
\hline 1 & $<4.5$ & 28.8 & $1923(30.0 \%)$ \\
2 & 4.5 to 7.5 & 26.2 & $2765(43.2 \%)$ \\
3 & 7.6 to 15.5 & 24.4 & $1505(23.5 \%)$ \\
4 & $>15.5$ & 21.4 & $205(3.2 \%)$ \\
\hline
\end{tabular}

P22

PB.22: Does the Nottingham predictor of bidimensional product of calcium accurately predict the success of wide local excision for ductal carcinoma in situ surgery in our centre?

R Gray, G Porter, I Steel

Plymouth Hospitals NHS Trust, Plymouth, UK

Breast Cancer Research 2013, 15(Suppl 1):P22

Introduction: A 2007 Nottingham paper suggests that a mammographic bidimensional product (BDP) of $<800 \mathrm{~mm}^{2}$ calcification in high-grade ductal carcinoma in situ (DCIS) and $<400 \mathrm{~mm}^{2}$ in low/intermediate DCIS is a powerful predictor of successful excision. We wanted to see whether their results were reproducible at our centre.

Methods: Eighty-four consecutive cases of DCIS over 18 months were reviewed and examined to determine the BDP of calcium (extent of mammographic calcification was measured in two orthogonal planes on the oblique view). Was reoperation required? Does the pathology report show clear margins? We excluded 15 patients that had a mastectomy (18\%).

Results: See Table 1.

Conclusion: We did not obtain similar results to the Nottingham paper. The success rate of WLE for low-grade DCIS was not (significantly) affected by BDP. Had BDP led to a recommendation not to perform WLE, the majority (75\%) of women in this low-risk group, most at risk of overtreatment, would have had a mastectomy when conservative surgery would have succeeded. While we have not the large numbers of the Nottingham paper, we feel that in our centre at least, BDP of calcification seems not to be a practically useful predictor of excision success in nonhigh-grade DCIS.

P23

PB.23: Effect of detector type on cancer detection in digital

mammography

A Mackenzie ${ }^{1,2^{*}}$, LM Warren ${ }^{1,2}$, DR Dance ${ }^{1,2}$, J Cooke ${ }^{3}$, M Halling-Brown $^{4}$, P Looney' ${ }^{1}$ DP Chakraborty ${ }^{5}$, MG Wallis ${ }^{6,7}$, RM Given-Wilson $^{8}$, KC Young $^{1,2}$ ${ }^{1}$ National Co-ordinating Centre for the Physics of Mammography, Royal Surrey County Hospital NHS Foundation Trust, Guildford, UK: ${ }^{2}$ Department of Physics, University of Surrey, Guildford, UK; ${ }^{3}$ Jarvis Breast Screening and Diagnostic Centre, Guildford, UK; ${ }^{4}$ Scientific Computing, Medical Physics, Royal Surrey County Hospital NHS Foundation Trust, Guildford, UK; ${ }^{5}$ Department of Radiology, University of Pittsburgh, PA, USA; ${ }^{6}$ Cambridge Breast Unit, Cambridge University Hospitals NHS Foundation Trust, Cambridge, UK; ${ }^{7} \mathrm{NIHR}$ Cambridge Biomedical Research Centre, Cambridge, UK; ${ }^{8}$ Department of Radiology, St George's Healthcare NHS Trust, London, UK Breast Cancer Research 2013, 15(Suppl 1):P23

Introduction: This work measured the effect that image quality associated with different detectors has on cancer detection in mammography using a novel method for changing the appearance of images.

Methods: A set of 270 mammography cases (one view, both breasts) was acquired using five Hologic Selenias and two Hologic Dimensions X-ray units: 80 normal, 80 with simulated inserted subtle calcification clusters, 80 with subtle real noncalcification malignant lesions and 30 with benign lesions (biopsy proven). These 270 cases (Arm 1) were converted to appear as if they had been acquired on two other imaging systems: needle image plate computed radiography (CR) (Arm 2) and powder phosphor CR (Arm 3). Three experienced mammography readers marked the location of suspected cancers in the images and

Table 1(abstract P22)

\begin{tabular}{lll}
\hline BDP & Successful WLE & Margins not clear \\
\hline High-grade DCIS & & \\
$<800 \mathrm{~mm}^{2}$ & $15,60 \%$ (Nottingham 69\%) & 10 \\
$>800 \mathrm{~mm}^{2}$ & $2,50 \%$ (Nottingham 24\%) & 2 \\
Low-grade DCIS & & \\
$<400 \mathrm{~mm}^{2}$ & $24,86 \%$ (Nottingham 73\%) & 4 \\
$>400 \mathrm{~mm}^{2}$ & $9,75 \%$ (Nottingham 33\%) & 3 \\
\hline
\end{tabular}


classified whether each lesion would require further investigation and the confidence in that decision. Performance was calculated as the area under curve (AUC) of the alternative free-response receiver operating characteristic curve.

Results: The AUCs of Arms 1 to 3 were $0.750,0.707$ and 0.648 for calcification clusters and $0.744,0.722$ and 0.694 for noncalcification cancers. The differences between all of the arms for calcification clusters were statistically significant $(P<0.05)$. No significant differences were found for noncalcification lesions. The percentage of correctly marked lesions that were classified as recalled lesions dropped from $58 \%$ to $39 \%$ (calcifications) and from $72 \%$ to $62 \%$ (non-calcifications) between Arms 1 and 3 .

Conclusion: Detector type has a significant impact on the detection and recall of calcification clusters but not noncalcification cancers.

\section{P24}

PB.24: Is digital breast tomosynthesis helpful within a symptomatic one-stop breast clinic for characterisation of subtle findings?

GJ Bansal', P Young, K Lim, Z Rees

University Hospital of Llandough, UK

Breast Cancer Research 2013, 15(Suppl 1):P24

Introduction: The study investigated the accuracy of two-view full-field digital (2D) mammograms (FFDM) by comparing its performance with twoview digital breast tomosynthesis (3D) plus FFDM combination in a symptomatic setting

Methods: A multi-case multi-reader study was conducted involving four imagers of varying experience. A total of 109 lesions from 103 patients who attended symptomatic breast clinics during a 7-month period between March 2012 and September 2012 were retrospectively read. All patients who had subtle signs on $2 \mathrm{D}$ images were retrospectively double read in a free response study and the findings recorded. The performance quality of the methods was evaluated using receiver operating characteristic (ROC) curves. Reader agreement ( $k$ values) was calculated and the area under curve (AUC) was compared between $2 \mathrm{D}$ imaging and $2 \mathrm{D}+3 \mathrm{D}$ imaging, regarding both readers and cases as random events.

Results: There was more inter-reader agreement between the two readers with $2 \mathrm{D}+3 \mathrm{D}$ combination $(k=0.391)$ compared with $2 \mathrm{D}$ images alone $(k=$ 0.153 ). In total, $85.19 \%$ of $M 3$ mammograms on $2 \mathrm{D}$ imaging were changed to $11.01 \% \mathrm{M} 3$ and $77.06 \% \mathrm{M} 2$ on combination of FFDM and DBT. There was more correlation between the mammographic scores and the final result for combination mode compared with $2 \mathrm{D}$ images alone $(P=0.0001)$. ROC analysis revealed the AUC for the combination (0.898) was significantly greater than 2D mammograms alone $(0.734 ; P=0.009)$.

Conclusion: DBT increases diagnostic sensitivity in a symptomatic setting and reduces the number of $\mathrm{M} 3$ mammograms, when used as an adjuvant to $2 \mathrm{D}$ images. Thus DBT has the potential of increasing work-flow efficiency in a symptomatic setting by reducing benign biopsies.

\section{P25}

PB.25: Relationship between volumetric breast density, age and hormonal factors

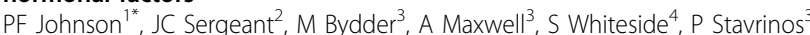
, M Wilson ${ }^{3}$, DG Evans, $s^{3,5}$, A Howell ${ }^{3,6}$, SM Astley ${ }^{2,3}$

${ }^{1}$ Uaniversity of Manchester Medical School, Manchester, UK; ${ }^{2}$ Centre for Imaging Sciences, University of Manchester, UK; ${ }^{3}$ Nightingale Centre and Genesis Prevention Centre, University Hospital of South Manchester, UK ${ }^{4}$ Department of Medical Statistics, University Hospital of South Manchester, UK; ${ }^{5}$ Department of Genetic Medicine, St Mary's Hospital, Manchester, UK Institute of Cancer Sciences, University of Manchester, UK

Breast Cancer Research 2013, 15(Suppl 1):P25

Introduction: Percentage breast density estimated visually or assessed by computer-assisted area-based measures declines with age, menopausa status and parity and increases with current HRT use. Automated volumetric density measurement methods including Quantra ${ }^{\mathrm{TM}}$ and Volpara ${ }^{\mathrm{TM}}$ remove subjectivity; it is important to determine how these methods relate to age and endocrine changes and here we describe these associations.

Methods: Women undergoing routine screening in the NHSBSP who agreed to enter the Predicting Risk Of Cancer At Screening (PROCAS) study completed questionnaires concerning personal information. Percentage volumetric breast density was measured using Quantra ${ }^{\mathrm{TM}}$ ( 21,370 women) and Volpara ${ }^{\mathrm{TM}}(11,023$ women), and WAS related to age and hormonal factors.

Results: Density significantly declined with age $(P<0.001)$ by both methods. In women aged $<50$ the mean density by Quantra ${ }^{\mathrm{TM}}$ was 19.23 for premenopausal and 16.29 for postmenopausal women $(P<0.05)$, and in women aged 51 to 55 years these figures were 18.06 and $15.72(P<0.05)$. For density measured by Volpara ${ }^{\mathrm{T}}$, the corresponding figures are 7.74 and 6.64 $(P<0.05)$ for women aged $<50$, and 7.54 and $6.00(P<0.05)$ for women aged 51 to 55 . Current HRT use was associated with significantly higher density $(P<0.05)$ for those aged over 50 (Volpara $\left.{ }^{\mathrm{TM}}\right)$ and from 51 to 70 (Quantra $^{\mathrm{TM}}$ ). Nulliparity was associated with higher breast density at all ages $(P<0.05$ for Quantra ${ }^{\mathrm{TM}}$ above the age of 50 and for Volpara ${ }^{\mathrm{TM}}$ from age 51 to 65 ).

Conclusion: These data indicate that percentage density by both volumetric methods is related to age and endocrine factors in the same directions as area-based methods. It is now important to determine their relationship to risk of developing cancer.

\section{P26}

PB.26: Significance of flat epithelial atypia at image guided breast biopsy

S Dani*, S Sudderuddin, G Ralleigh, N Zaman, A Gupta, N Barrett,

D Cunningham, B Faissola, S Comitis, W Svensson, A Lim, R Williamson, $\checkmark$ Stewart

Imperial College Healthcare NHS Trust, London, UK

Breast Cancer Research 2013, 15(Suppl 1):P26

Introduction: The term flat epithelial atypia (FEA) was introduced in 2003 by the WHO working group on the Pathology \& Genetics committee of tumours of the breast. Previously known as columnar cell change with atypia, these lesions usually present as microcalcification and although there is some evidence to suggest that FEA represents a precursor lesion in the spectrum of low-grade breast neoplasia, there are limited data concerning the ultimate clinical impact and optimum management strategy. The aim of this study was to determine the radiological characteristics of FEA and final histology following surgical excision at our centre.

Methods: Retrospective review of 950 consecutive image-guided core and vacuum biopsies performed over an 8-year period revealed 162 lesions with FEA as the most prominent pathologic entity; concomitant lobular neoplasia, $\mathrm{ADH}$ or malignancies were excluded. The radiologic characteristics of FEA/ CCC lesions were assessed with respect to size, morphology and multifocality at presentation. Biopsy technique and final histology of these lesions following surgical excision were recorded.

Results: The rate of upgrade to a more significant pathology was $10 \%$ (on provisional data, complete results will be presented) with significant pathology being DCIS of varying grade. Microcalcification was the most common mammographic sign.

Conclusion: Surgical excision of FEA at our centre has resulted in a low rate of upgrade to malignancy supporting current thinking that some lesions may be managed by vacuum excision, thus reducing the surgical diagnostic excision rate.

\section{P28}

PB.28: Is second-time vacuum-assisted biopsy effective in improving preoperative diagnosis rate of screen-detected DCIS?

S Pattison*, A Kumar, W Teh, R Patel

NLBSS, Edgware, UK

Breast Cancer Research 2013, 15(Suppl 1):P28

Introduction: The NHSBSP standards for non-operative diagnosis are a target of $95 \%$ for invasive cancers (minimum $90 \%$ ) and $90 \%$ for non-invasive cancer (minimum 85\%). The lower target for non-invasive cancers reflects the difficulty in achieving definitive diagnosis of DCIS. Our unit has routinely used vacuum biopsies (VAB) for non-operative diagnosis of microcalcifications. Following an audit in 2009, repeat VAB were extended to B3 cases selected in MDT in order to improve non-operative diagnosis of DCIS.

Methods: KC 62 report was used to identify all screen-detected cancers in 2011 and 2012. The mammographic characteristics, type and number of biopsy procedures and results were reviewed and correlated against final 
surgical outcomes. A final outcome of LCIS was excluded from the audit. The results were compared against a similar audit in 2008 and 2009.

Results: In total, $86 / 330$ (26.1\%) were DCIS. A total of $90.7 \%$ (78/86) of noninvasive cancers were diagnosed preoperatively compared with $86 \%$ in 2008 and 2009. Non-operative diagnosis was achieved in $81.4 \%(70 / 86)$ on the first biopsy and $9.3 \%(8 / 86)$ on the second biopsy. Four cases of DCIS were completely excised by VAB. Six cases upgraded by repeat VAB were microcalcifications. Seven of eight cases of DCIS with no preoperative diagnosis were not subjected to second-time $V A B$.

Conclusion: Second-time $V A B$ successfully improve non-operative diagnosis of DCIS even when VAB are routinely used in the assessment of microcalcifications. The practice should be considered for all B3 cases in order to further improve the non-operative diagnosis rate for DCIS.

P29

PB.29: Ultrasound-guided vacuum-assisted percutaneous excision of breast papillomas: results of long-term follow-up

AJ Maxwell ${ }^{1,2^{*}}$, G Mataka ${ }^{1,3}$, S Whiteside ${ }^{4}$

${ }^{1}$ Royal Bolton Hospital, Bolton, UK; ${ }^{2}$ Nightingale Centre and Genesis

Prevention Centre, University Hospital of South Manchester, UK; ${ }^{3}$ Breast Unit,

Royal Lancaster Infirmary, Lancaster, UK; ${ }^{4}$ Department of Medical Statistics,

University Hospital of South Manchester, UK

Breast Cancer Research 2013, 15(Suppl 1):P29

Introduction: The purpose was to determine the frequency of recurrent/ residual papillomas in women who had previously undergone vacuumassisted excision biopsy (VAB) of benign papillomas without atypia and to identify factors that may be associated with recurrence.

Methods: Women who had undergone VAB of papillomas and had subsequent breast imaging were identified from hospital records. Papilloma size, VAB device used and number of cores was recorded and subsequent imaging reviewed. Possible associations between the likelihood of recurrent/ residual papilloma and patient age, lesion size and excised volume to papilloma volume ratio were analysed using Mann-Whitney $\mathrm{U}$ tests.

Results: Thirty-four women had subsequent imaging available. Median follow-up was 1,052 days. Twelve women (35\%) had evidence of a mass at the excision site, with a median time to identification of recurrent/residual papilloma of 1,369 days. In three of the six cases that underwent ultrasound the recurrence was larger than the original lesion. Three recurrences were removed at open surgical biopsy and one by further VAB. None of the 34 women were diagnosed with atypia or malignancy during follow up. No significant association was found between the risk of recurrence and the age of the patient, the size of the initial lesion or the ratio of the volume of tissue removed to the lesion volume.

Conclusion: Recurrent/residual papillomas are common but most do not require further intervention. No definite risk factors were identified. VAB remains the excision method of choice for most benign papillomas without atypia.

P30

PB.30: What is your biopsy threshold in symptomatic clinic and what should it be? B2:B5 biopsy ratio: 18 months in our centre

R Gray, G Porter, J Steel

Plymouth Hospitals NHS Trust, Plymouth, UK

Breast Cancer Research 2013, 15(Suppl 1):P30

Introduction: While a $<2 \%$ benign biopsy rate is widely regarded as the gold standard in the screening population, there is no such guidance for biopsy in symptomatic patients. When symptomatic patients present with a lump visible on ultrasound, the decision to biopsy is down to the individual radiologist. There is currently no gold standard for the benign: malignant biopsy ratio.

Methods: A prospective list of all symptomatic clinic biopsies was compiled over 18 months from January 2012 to May 2013. Imager and histological outcome of each biopsy (B1 to B5) were recorded. Cancer detection rate for each operator per 1,000 symptomatic cases was also calculated. Axillary biopsies were excluded.

Results: Six anonymised operator biopsy results were recorded. There is a wide variation with our centre of $\mathrm{B} 2 \mathrm{~B} 5$ ratios without significant variation in the cancer detection rate between operators.
Conclusion: This study demonstrates considerable variation in biopsy threshold within one unit. There is no correlation of biopsy rate and cancer detection in this dataset, meaning that potentially there are many unnecessary biopsies performed. This study has stimulated discussion in our unit, and is a useful and simple audit to perform to investigate biopsy threshold. If performed by a number of units nationally, it may be possible to assess the range for these parameters and to suggest a normal range.

\section{P31}

PB.31: B3 lesions and vacuum-assisted biopsy: a national survey to gauge current practice

CL Strachan*, A Shaaban, K Horgan, N Sharma

St James University Hospital, Leeds, UK

Breast Cancer Research 2013, 15(Suppl 1):P31

Introduction: Breast screening detects asymptomatic abnormalities which occasionally on biopsy are classified indeterminate (B3). Such lesions have malignant potential and traditionally are subject to open diagnostic excision biopsy. Vacuum-assisted biopsy (VAB) offers larger representative tissue sampling, and may act as a therapeutic measure completely excising the lesion. The use of VAB in the NHSBSP varies widely. Currently there are no relevant national guidelines to streamline practice.

Methods: A survey was sent to 80 screening units throughout England, comprising seven questions concerning the use of $\mathrm{VAB}$ for $\mathrm{B} 3$ lesions.

Results: Fifty-four responses (67.5\%) were received. Twenty-two per cent of units do not perform VAB, $55 \%$ perform first-line and $77.8 \%$ second-line VAB. For B3 lesions without atypia, $68 \%$ would proceed to second-line $V A B$ whilst $25 \%$ advocate open diagnostic excision following initial (14G) core. Management of B3 lesions with atypia was more discordant, with the majority of units opting for second-line VAB for FEA, ALH and LCIS, and second-line diagnostic excision for radial scars, ADH (atypical intraductal proliferation) and papillomas with atypia. Following first-line $V A B$, most units would proceed to diagnostic excision rather than second-line VAB.

Conclusion: Management of B3 lesions varies significantly across screening units. There is no concordance in the use of VAB for diagnosis or management of $B 3$ lesions. Whilst there is a trend toward second-line VAB for atypias, significant numbers still opt for diagnostic excision. Consensual national guidelines to standardise and guide management would provide equity of care for this difficult management entity.

\section{P32}

PB.32: Does vacuum-assisted biopsy decrease the B3 rate in stereotactic biopsy of breast lesions?

T Seaton", S Khan, V Stewart, G Ralliegh, N Zaman, N Barrett, S Comitis,

A Gupta, W Svensson, A Lim, R Williamson, D Cunningham

Charing Cross Hospital, West London Breast Screening Service, London, UK

Breast Cancer Research 2013, 15(Suppl 1):P32

Introduction: Vacuum-assisted biopsy (VAB) systems have been shown to outperform $14 \mathrm{G}$ core needle biopsy (CNB) reducing the need for diagnostic or multiple surgeries. We introduced VAB in 2011 with the aim of reducing our B3 rate and increasing rate of preoperative diagnosis of invasive cancer. Methods: Mammographic abnormalities requiring stereotactic biopsy in a defined period which had either $14 \mathrm{G}$ CNB or VAB were included. Data collated included mammographic findings, biopsy and surgical outcome.

Results: Ninety 14G-CNBs (November 2010 to May 2011) were compared with 78 VABS (July 2012 to January 2013). There was no reduction in our B3 rate (12 and 13 cases respectively) although $V A B$ had higher sensitivity with better correlation between biopsy diagnosis and surgical histology $(77 \%$ compared with 42\%). Four of 12 (25\%) B3 lesions diagnosed following $14 \mathrm{G}$ CNB were upgraded to DCIS following surgical excision compared with no cases following VAB. Preoperative invasive malignancy diagnostic rates were unchanged, $15 \%$ of cases were upgraded in both groups.

Conclusion: VAB has not reduced our B3 rate but has increased the accuracy of our preoperative diagnosis of non-invasive cancer supporting current theories that patients who have undergone vacuum-assisted biopsy may not require surgical diagnostic excision. However, we recognise the number of cases is low and further cases will be analysed and presented. 
P33

PB.33: Vacuum-assisted biopsy for lesions of uncertain malignant potential: further evidence of its suitability as a safe alternative to surgery D Tzias*, B Bhaludin, LS Wilkinson, S George, H Gay

St George's Hospital and South West London Breast Screening Service, London, UK

Breast Cancer Research 2013, 15(Suppl 1):P33

Introduction: Increasing evidence supports vacuum-assisted biopsy (VAB) instead of surgical excision for the management of lesions of uncertain malignant potential (B3). This followed reports of up to $34 \%$ upgrade to malignancy on excision. In January 2012, regional guidance was adopted to clarify the options for managing such lesions. In our department, we have used similar local guidelines since 2011. We report our analysis of the management of B3 lesions before and after the introduction of VAB.

Methods: All B3 lesions were identified retrospectively, using our screening database, before (1 January 2008 to 31 December 2009) and after (1 January 2011 to 31 December 2012) VAB use. Final pathological diagnosis following surgical excision or VAB was recorded for both groups. The findings of annual surveillance mammography were also recorded for lesions which remained $B 3$ but were radiologically excised following $V A B$.

Results: Before the use of VAB there were $94 \mathrm{~B} 3$ lesions, 80 (85\%) of which underwent surgical excision. After the use of $\mathrm{VAB}$ there were $85 \mathrm{~B} 3$ lesions, of which $17(20 \%)$ went on to have diagnostic surgery. The percentage of upgrade to malignancy following further sampling was equal in both groups, at $18 \%$. Sixteen of 26 lesions, which remained B3 following VAB excision, had surveillance mammography, none of which demonstrated suspicious findings.

Conclusion: Our study provides further evidence that VAB is a safe alternative to surgery for the management of B3 lesions, with identical upgrade rates and reassuring follow-up results, following its use in our department.

References

1. Rajan S, Shaaban AM, Dall BJ, Sharma N: New patient pathway using vacuum-assisted biopsy reduces diagnostic surgery for B3 lesions. Clin Radiol 2012, 67(3):244-9.

2. Tennant SL, Evans A, Hamilton $\amalg$, James J, Lee AH, Hodi Z, Ellis 10 , Rakha EA, Wilson AR: Vacuum-assisted excision of breast lesions of uncertain malignant potential (B3) - an alternative to surgery in selected cases. Breast 2008, 17(6):546-9.

3. Lieske B, Ravichandran D, Alvi A, Lawrence DA, Wright DJ: Screen-detected breast lesions with an indeterminate (B3) core needle biopsy should be excised. Eur J Surg Oncol 2008, 34(12):1293-8.

4. Wilkinson LS, Wells C, Teh W, Desai A, Wilson R: The management of

indeterminate breast lesions - a clinicians guide. London region quality assurance reference centre. Guidance on management of indeterminate breast lesions 2012.

\section{P34}

PB.34: Results by letter for low-risk breast biopsies: an audit of current practice at Nottingham Breast Institute

WK Al-Obaydi", EJ Cornford, SL Tennant

Nottingham Breast Institute, City Hospital, Nottingham, UK

Breast Cancer Research 2013, 15(Suppl 1):P34

Introduction: Patients undergoing breast biopsy are usually asked to return to the results clinic, to enable face-to-face counselling and support. However, a significant proportion of biopsies yield benign or normal results. Since 2011 we have offered the choice of results by letter to selected patients undergoing breast biopsy. This is at the discretion of the assessing radiologist or surgeon, and includes radiologically guided biopsy of benign/ probably benign lesions and clinical biopsies where the imaging is normal. Our aim was to identify our accuracy in assessing a patient as suitable for results by letter.

Methods: Retrospective review of 100 consecutive patients entering this pathway between January and May 2013. Patients were identified from MDT lists, and hospital RIS and pathology systems were interrogated.

Results: One hundred females, age range 20 to 79 years (mean 42), were included. Eighty-nine presented symptomatically, 11 were recalled from screening. Ninety-two core biopsies and eight fine needle aspirates (FNAs) were performed under ultrasound guidance (91 patients), stereotactically (two patients) or freehand (seven patients with normal imaging). Cytology/ histology was normal or benign in 98 patients. Two patients were recalled following FNA results of $\mathrm{C} 4$ and $\mathrm{C} 5$ and were subsequently diagnosed with DCIS and invasive cancer, respectively.

Conclusion: Our current practice of identifying low-risk patients is $98 \%$ accurate. Sending selected results by letter can obviate the need for a results clinic appointment, with benefits to both patients and staff. However, a robust method of tracking these patients is vital, as well as thorough MDT discussion.

\section{P35}

PB.35: Comparison of large-bore vacuum-assisted biopsy and surgical diagnostic excision biopsy in B3 breast lesions

AH Tang*, ND Forester

Department of Breast Radiology, Royal Victoria Infirmary, Newcastle, UK

Breast Cancer Research 2013, 15(Suppl 1):P35

Introduction: B3 lesion management is unclear. Despite varying malignancy risk, diagnostic excision was conventional, revealing malignancy in some and benign features in others. Large-bore vacuum-assisted biopsy (VAB, 7 to 8G) can enhance pathological certainty, providing reassurance of benignity, or identify co-existing malignancy, with many groups moving towards VAB to replace surgical excision biopsy. However, little research has addressed the accuracy of VAB compared with surgical biopsy in this situation.

Methods: From November 2011 to May 2013, we incorporated VAB into the management of all B3 lesions identified by 10/14G biopsy. Excision biopsy was still performed on any lesion initially identified as B3 with atypia, unless upgraded by $V A B$. The $V A B$ result and surgical excision outcomes were compared.

Results: A total of 181 lesions were identified and considered for VAB. Atypia was present in 102 lesions. Sixty-five of 102 lesions had VAB, with 11 upgraded to malignancy. Of the $54 / 65$ lesions not upgraded by VAB, 25 proceeded to excision biopsy, revealing DCIS in seven. A total 37/102 lesions did not have VAB (fibro-epithelial lesions/technical factors); malignancy was identified in five at excision biopsy. The overall malignancy rate was $23 / 102$ (22.5\%). Of $\mathrm{VAB} / \mathrm{excision}$ biopsy discordant lesions, average tissue obtained at $V A B$ was $2.4 \mathrm{~g}$, compared with $2.9 \mathrm{~g}$ in concordant lesions (NS).

Conclusion: VAB can increase preoperative detection of malignancy, but has an associated miss rate. Thirty per cent of the DCIS present in B3 lesions with atypia was missed by second-line VAB. This is important to appreciate as women are placed on surveillance programmes for B3 lesions following $\mathrm{VAB}$, but without completion excision biopsy.

P36

PB.36: Double reporting of patients discharged from the assessment clinic: the North Lancashire and South Cumbria experience G Mataka ${ }^{1,2}$

'University Hospitals of Morecambe Bay NHS Foundation Trust, Lancaster, UK; ${ }^{2}$ North Lancashire and South Cumbria Breast Screening Programme, Lancaster, UK

Breast Cancer Research 2013, 15(Suppl 1):P36

Introduction: There are currently no available guidelines regarding the double reporting of patients discharged from the assessment clinic back to the screening programme.

Methods: A retrospective audit of patients who were referred for arbitration following discordant double reporting of patients discharged from the assessment clinic was performed. This was performed over a 2-year period from May 2011 to May 2013. The available imaging was reviewed. The outcome of arbitration was documented. The morphology of the lesion, whether a second assessment was required and outcome of the second assessment were also recorded.

Results: A total of 4,445 patients were assessed in our breast unit. All patients discharged back to the screening programme following assessment were double reported at the end of the clinic. Thirteen $(0.29 \%)$ patients were referred for arbitration. The morphology of the lesions was as follows: asymmetric density $(n=8)$, microcalcification $(n=4)$ and distortion $(n=1)$. Following arbitration, nine patients were discharged back to the screening programme. The remaining four patients underwent a second assessment. All patients who underwent a second assessment 
had a biopsy performed. Three of these patients were discharged following a benign biopsy result. The remaining patient was diagnosed with a grade I invasive ductal carcinoma.

Conclusion: The number of patients requiring a second assessment is very small $(0.09 \%)$, limiting the psychological impact on our patients. Asymmetric densities are the most common abnormality resulting in referral for arbitration. Double reporting of assessment clinic patients discharged back to the screening programme is now standard practice in our unit.

\section{P37}

PB.37: Screen-detected, noncalcified, mammographic lesions with normal or benign ultrasound findings: is stereotactic biopsy necessary? D Tzias", S Yusuf, L Wilkinson

St George's Hospital and South West London Breast Screening Service, London, UK

Breast Cancer Research 2013, 15(Suppl 1):P37

Introduction: Ultrasound has long been used in the symptomatic service, not only to distinguish cystic from solid masses but also to help in the differentiation of benign from malignant lesions. The ability to correlate a benign ultrasound mass with a mammographic mass eliminates the need for further intervention. We evaluate the need for stereotactic biopsy in screen-detected, nonpalpable lesions without calcification, which have either benign or normal sonographic findings.

Methods: Patients who had stereotactic biopsy for mammographic lesions from January 2011 to January 2013 were retrospectively identified from our screening database. Clinical examination and ultrasound findings, presence of calcification and pathological diagnosis were recorded. Final imaging opinion was also recorded from the pathology request forms.

Results: Of 4,339 patients recalled for assessment, 1,860 had a biopsy (853 stereotactic and 1,007 ultrasound guided). Stereotactic biopsies were for microcalcification $(n=748)$ and for 105 impalpable, noncalcified densities with normal $(n=73)$ or benign $(n=32)$ ultrasound findings. Malignancy was detected in eight $(8 \%)$ noncalcified lesions and $169(23 \%)$ microcalcifications $(P<0.0002$, Fischer exact test). Simple cysts were detected in $28 / 32$ (88\%) of cases with benign ultrasound findings. Suspicion of malignancy was mentioned in 38/105 (36\%) final imaging opinions. Asymmetry $(n=4)$ and distortion $(n=2)$ were the commonest lesion features associated with a positive biopsy result.

Conclusion: Stereotactic biopsy for screen-detected mammographic densities with normal or benign ultrasound findings has a low yield of malignancy. Careful analysis of mammographic findings, ultrasound correlation and further multidisciplinary discussion could help reduce unnecessary biopsies.

References

1. Stavros AT, Thickman D, Rapp CL, Dennis MA, Parker SH, Sisney GA: Solid breast nodules: use of sonography to distinguish between benign and malignant lesions. Radiology 1995, 196(1):123-134.

2. Lucas-Fehm L: Sonographic mammographic correlation. Applied Radiology 2005, 34(2):20

\section{P38}

PB.38: In the context of overdiagnosis, does size matter?

S Bhuva*, I Haigh, M McMahon, B Dall, D Dodwell, N Sharma

Leeds Teaching Hospitals, Leeds, UK

Breast Cancer Research 2013, 15(Suppl 1):P38

Introduction: The Marmot Review showed that although breast screening saves lives, it is harmful through overdiagnosis - treating cancers that would not otherwise have ever become clinically apparent. Currently, there is no size threshold for recalling screening patients with calcifications. Our aim was to assess whether a minimum size threshold would reduce overdiagnosis.

Methods: We conducted a retrospective review of 375 screening patients with microcalcifications over 24 months. We assessed all patients with pure calcifications $\leq 10 \mathrm{~mm}$ documenting core biopsy, final histology and treatment.

Results: Sixty-one cases of microcalcifications $\leq 10 \mathrm{~mm}$ : eight benign, 40 in situ cancers and 13 invasive cancers. This group was subcategorised into calcifications: $0 \leq 5 \mathrm{~mm}$ ( 24 patients) and $5 \leq 10 \mathrm{~mm}$ (37 patients). In the 0 $\leq 5 \mathrm{~mm}$ group, there were 16 in situ (low-grade, one; intermediate grade, seven; high grade, eight) and two invasive cancers (G2 ductals ER/PR ${ }^{+} \mathrm{Her} 2$ node-negative). In the $5 \leq 10 \mathrm{~mm}$ group, there were 24 in situ (low-grade, three; intermediate grade, 12; high grade, nine) and 11 invasive cancers (four G1ER ${ }^{+} \mathrm{Her} 2^{-}$node-negative, six $\mathrm{G}^{2} \mathrm{ER}^{+} \mathrm{Her2}{ }^{-}$, one triple-negative). One of these six cases was node-positive (micrometastasis) and one $\mathrm{G} \mathrm{ERPR}^{+} \mathrm{Her} 2^{-}$node-negative. All underwent wide local excision, and all but one patient with invasive carcinoma received radiotherapy.

Conclusion: Recalling focal clusters of microcalcifications $(<10 \mathrm{~mm})$ identified a high rate of cancers: $66 \%(40 / 61)$ in situ and $21 \%(13 / 61)$ invasive. With regards to overdiagnosis: $51 \%(27 / 53)$ of cancers were low/ intermediate-grade DCIS or G1 invasive and $49 \%$ (26/53) were high-grade DCIS or invasive $\mathrm{G} 2 / 3$. Therefore size is not a key factor in reducing overdiagnosis.

P39

PB.39: Minimising the impact of breast screening extension: a 1-year experience of a South West breast screening unit K Giles $^{1^{*}}$, R Currie , $^{1,2}$

${ }^{1}$ Royal Devon and Exeter Hospital, Exeter, UK; ${ }^{2}$ Exeter and North Devon Breast Screening Unit, Exeter, UK Breast Cancer Research 2013, 15(Suppl 1):P39

Introduction: In 2007, the Cancer Reform Strategy announced an extension to the NHS Breast Screening Programme (NHSBSP) from women aged 50 to 70 to those 47 to 73 years. Pilot studies involved a 50\% randomised cohort at either the upper or lower end of the extension. We detail how our BSU has instigated the complete lower age extension largely within existing capacity.

Methods: Data collection from NBSS. Working practices identified from local policy.

Results: Screening numbers increased from 22,022 to 26,272 (a $19 \%$ increase), generating an extra 355.2 screening hours. This constituted a $76 \%$ invite uptake in women aged 47 to 49 . Screening appointments were reduced from 6 to 5 minutes. Mammographers working patterns - but not overall hours - were changed. An extra workstation was purchased for additional film reading by existing personnel. All assessment clinics became fully booked sessions (recall rate 9.4\%). Biopsies were largely undertaken in existing capacity but provision was increased when needed by altering staff working patterns. Women requiring MDT discussion led to a marginal increase in MDT length. All biopsy results were delivered by breast care nurses - the extra work load required an additional session (4 hours) to fulfil the demand. All screening targets were met.

Conclusion: Full lower end age extension has undoubtedly increased our screening work load. However, by reducing the screening appointment time, altering working patterns and purchasing a single extra workstation, the impact to the service has been minimised. Potential difficulties do arise in the event of sickness or leave.

\section{P40}

PB.40: Clinical recall audit: are we following national guidelines?

F Kilburn-Toppin*, S Karia, M Wallis

Addenbrooke's Hospital, Cambridge, UK

Breast Cancer Research 2013, 15(Suppl 1):P40

Introduction: Women with normal mammograms may be recalled from the NHSBSP if they indicate significant clinical symptoms at the time of screening. It is important that mechanisms are in place to record clinical abnormalities, with a clear written protocol.

Methods: The Cambridgeshire screening population was examined from April 2009 to April 2012. Our standard was that all patients with clinical symptoms should have a clinical alert on NBSS, in keeping with 2005 assessment guidelines [1]. We analysed the number of women who were clinically recalled for this period, if a clinical alert was entered on NBSS, the symptoms underlying the clinical recall, and the clinical outcome.

Results: A total of 50,785 patients were screened, of which 165 with normal mammography were placed on clinical recall. In total, 151 (92\%) had a clinical alert on NBSS. Thirty-seven of these had an abnormality detected, of which the majority $(n=17)$ were cysts. Four ( $2 \%$ or 0.8 per 10,000 screened) had an invasive malignancy. A palpable lump was the commonest clinical 
symptom for recall, but also included nipple discharge, pain and patient anxiety.

Conclusion: Electronic NBSS documentation for clinical recall is an important measure to ensure patients with normal mammograms but clinically significant symptoms are not returned to routine screening. Radiographers should receive appropriate training to recognise significant breast symptoms, with a clear written protocol for clinical recall. New local guidelines have been implemented prior to re-audit.

Reference

1. Quality Assurance Guidelines for Breast Cancer Screening Radiology: NHSBSP Publication No 59. NHS Breast Screening Programme. 2005.

\section{P41}

PB.41: Audit of screening-detected breast cancers with discordant interpretations on double-read screening mammography

B Batohi", R Rahim, M Adejolu, M Michell

King's College Hospital, London, UK

Breast Cancer Research 2013, 15(Suppl 1):P41

Introduction: Discordant breast cancers are identified following differing interpretations on double-read screening mammograms. The aim of this audit was to analyse the mammographic features of discordant breast cancers to improve reader sensitivity.

Methods: The screening programme database was used to identify al breast cancers that were diagnosed from 1 April 2010 to 31 March 2012. Discordant cases were reviewed at a consensus meeting in which the mammographic sign, size, site, parenchymal pattern and final histology were recorded.

Results: Fifty-seven of a total of 670 cancers had discordant reads. The most common mammographic abnormality was spiculated masses (30\%). Microcalcifications accounted for $25 \%$ of cases. Eighty-five per cent of the abnormalities were small (i.e. $\leq 15 \mathrm{~mm}$ ). No single view was noted to be more likely to reveal the abnormality. The histology results showed no difference in distribution of tumour type compared with nondiscordant tumours. The second reader identified $69 \%$ of the discordant cancers.

Conclusion: A total $8.5 \%$ of the screen-detected cancers in this audit were discordant-read cancers, which corresponds with published findings [1]. The mammographic features of discordant cancers were similar to nondiscordant cancers. The study supports the continued use of two readers to maximise sensitivity [2].

References

1. Cornford EJ, et al: The pathological and radiological features of screendetected breast cancers diagnosed following arbitration of discordant double reading opinions. Clin Radiol 2005, 60:1182-1187.

2. Hackshaw AK, Wald NJ, Michell MJ, Field S, Wilson AR: An investigation into why two-view mammography is better than one-view in breast cancer screening. Clin Radiol 2000, 55:454-458.

\section{P42}

PB.42: Arbitration of round masses: understanding the variability of recall rates

AL Wharton", CG Taylor, JC Cooke

Jarvis Breast Screening Centre, Guildford, UK

Breast Cancer Research 2013, 15(Suppl 1):P42

Introduction: It is important to keep recall rates to a minimum. At the Jarvis Centre daily arbitration is performed by two experienced film readers, a regular pair each weekday. Observation that different arbitration pairs have varying thresholds for recalling round masses prompted this audit.

Methods: Thirty-six digital cases with round masses were selected during arbitration over a 1-month period. All cases were anonymised and rearbitrated by six pairs of arbitrators who usually work together. The pairs included at least one film reader with over 10 years experience. Specific mammographic features and the final audit recall decision were recorded.

Results: A total $17 / 36(47 \%)$ cases were recalled at the original arbitration. Arbitration pairs had different audit recall rates ranging from 39 to $81 \%$. All arbitrators agreed to either rescreen or recall in $1 / 3$ of cases. Results showed there was variation in both perception of mammographic features present and the importance given to other factors such as age, multiplicity, lesion location and density; affecting recall even in the presence of benign mammographic features.

Conclusion: There is considerable variation in opinion between arbitration pairs due to the complex nature of interpreting which mammographic features are present and balancing these against other factors such as age, multiplicity, lesion location, and density. It is useful to reflect on the steps in the decision-making process to understand the science behind the art in an attempt to achieve more consistency between pairs and keep recall rates down.

\section{P43}

PB.43: Review of the determinants of poor screening uptake at City, Sandwell and Walsall Breast Screening Units and the steps taken to improve attendance

H Khan ${ }^{1,2^{*}}$, S Meraj ${ }^{1}$, A Wilbraham ${ }^{1,2}$, D Cox ${ }^{1,2}$, R Bhatt $^{1,2}$, J Yates $^{1,2}$,

J Waldron ${ }^{1,2}$, A Powell ${ }^{1,2}$

${ }^{1}$ Sandwell and West Birmingham NHS Trusts, Birmingham, UK; ${ }^{2}$ Walsall

Manor Hospital, Walsall, UK

Breast Cancer Research 2013, 15(Suppl 1):P43

Introduction: The aim of National Health Services Breast Screening Programme (NHSBSP) is to reduce morbidity and mortality related to breast cancer. The success of NHSBSP depends upon a high proportion of women attending for their 3-yearly screening mammograms. The national target for screening attendance is $80 \%$ and the minimum standard is $70 \%$. For 2010 and 2011, an overall national screening uptake for women aged 50 to 70 was $73.4 \%$. The overall uptake rate for our screening service is recorded as the fifth lowest in achieving minimum uptake targets. The purpose of this review was to analyse the factors associated with poor uptake in some of our screening regions and assess the effectiveness of the methods used to maximise attendance in these regions.

Methods: The source of our data collection was west Midlands Cancer Intelligence Unit and NHSBSP database. We analysed the trends in screening uptake from 2007 to 2013.

Results: Social deprivation, population size, ethnicity, availability and access, and education and awareness are some of the most significant factors that affect uptake. Interventions were devised to target these factors. The initiatives used were simple such as patient letters, promotional posters and multi-lingual leaflets, DNA flyers, telephonic reminders and most importantly collaborative work with GP surgeries. The results were encouraging and we managed to increase the prevalent uptake by $7.87 \%$ in our lowest uptake region.

References

1. NHS Breast Screening Programme Annual Review 2012, ISBN 978-1-84463093-6.

2. NHS Cancer Screening Programme 2005, ISBN 1844630242 | Archived.

3. Maximizing Screening Attendance Reference Guide 2009, Dr Christiane Zelenyanszki Joint Screening Lead. NWLCN (North West London Cancer Network) December 2009.

P44

PB.44: Audit of interval cancers from family history breast screening R Dunn ${ }^{* *}$, S Astley $^{1}$, M Bydder $^{2}$, DG Evans ${ }^{1,2}$, A Howell ${ }^{1,2}$, J Sergeant ${ }^{1,2}$, AJ Maxwell ${ }^{2}$

${ }^{1}$ University of Manchester, UK; ${ }^{2}$ Nightingale Centre and Genesis Prevention Centre, University Hospital of South Manchester, UK

Breast Cancer Research 2013, 15(Suppl 1):P44

Introduction: A total of 267 women screened by the family history clinic (FHC) have been diagnosed with breast cancer since 1990. In 73 women the cancers were diagnosed in the interval between mammographic screens, which were performed every 1 to 2 years and single read. The aim of this audit was to compare performance with that of the NHS Breast Screening Programme (NHSBSP).

Methods: Screening mammograms were found for 21 women, who had 22 interval cancers (one was bilateral). Thirteen had symptomatic mammograms available for comparison. The site of the cancer was known for eight of the remaining nine cases. Blinded review of the screening mammograms was performed and then comparison was made with the symptomatic mammograms or other available location data. 
Results: The screening mammograms were classified as follows: Category 0 (no symptomatic mammograms available), 9; Category 1 (screening mammograms normal), 7; Category 2A (subtle appearances, recall not justified), 2; Category 2B (subtle appearances, recall indicated), 2; and Category 3 (suspicious abnormality), 2. Of the eight Category 0 cases with a known tumour location, a subtle abnormality was visible in this area in one case but the remainder were normal. Thus the screening mammograms were normal in 14 of the 21 cases $(67 \%)$ where the tumour location was known. Fifteen (68\%) of the interval cancers were diagnosed within 12 months of the last mammogram.

Conclusion: The proportion of Category 2 and 3 cases is similar to that seen in the NHSBSP. Double reading may reduce the number of these in the FHC population.

P45

PB.45: Evaluation of a pilot MRI breast surveillance project for young women at high risk of breast cancer

S De Souza ${ }^{1 *}$, N Evans' ${ }^{1}$, R Fox $^{1}$, A Murray $^{2}$

${ }^{1}$ Screening Division, Public Health Wales, Cardiff, UK; ${ }^{2}$ All Wales Medical Genetics Service, Cardiff, UK

Breast Cancer Research 2013, 15(Suppl 1):P45

Introduction: NICE Clinical Guidelines recommend that young women at high risk of breast cancer are offered annual surveillance with MRI and digital mammography. A feasibility study was run over 3 years (2010 to 2013) for women with known BRCA1 or BRCA2 mutations and those with, or at high risk of, a TP53 gene mutation. We aimed to explore the practicalities of delivering a surveillance service for these high-risk women.

Method: Eligible women were identified by the All-Wales Medical Genetics Service and offered annual MRI, with digital mammography where appropriate, at three centres across Wales. Administration was managed by Breast Test Wales.

Results: Qualitative feedback showed that women were willing to travel for appointments and the service was acceptable. Only 12 of the 38 participants attended all years. This is a dynamic group - some develop cancer, others opt for risk-reducing surgery, and each year some were ineligible as they were pregnant or breastfeeding. Administration of the system was complex, involving different professional groups working across sites. Standards for waiting times for results were not met. Few radiologists in Wales meet the English standard of 100 breast MRI examinations per year to be eligible as readers. This poses a challenge for service delivery. MRI scanner capacity is also limited.

Conclusion: The enthusiasm of the multidisciplinary team ensured the project was successful and the service delivered. However, there were major administrative and resource challenges, and the pilot service model was unsustainable. The evaluation will be used to inform decisions on future service provision.

P46

PB.46: Peutz-Jeghers Syndrome and carcinoma of the breast: call for new breast imaging surveillance guidelines

FJ Tsang*, S Mallappa, W Teh, SK Clark

Northwick Park and St Mark's Hospitals, Northwest London Hospitals NHS

Trust, London, UK

Breast Cancer Research 2013, 15(Suppl 1):P46

Introduction: Peutz-Jeghers Syndrome (PJS) is a rare autosomal dominantly inherited polyposis syndrome caused by STK11 germline mutation. PJS is characterised by gastrointestinal polyps and mucocutaneous pigmentation. These patients have an increased risk of developing cancers, luminal gastrointestinal cancers and breast cancer being the most common, then pancreatic cancer $[1,2]$.

Methods: $A$ retrospective review of a prospectively maintained database at a tertiary referral centre was carried out. A total of 136 patients from 92 families were included.

Results: The median age when patients were first seen was 20 years (range, 2 to 65 ). Twenty-seven carcinomas were detected in 19 patients; 15 were carcinoma of the breast (55\%) (two patients had bilateral disease). All of the breast cancers were in women and were predominantly ductal carcinoma in situ. Of the 13 patients with breast cancer, a STK11 mutation was detected in eight (62\%).

Conclusion: The cumulative risk of developing breast cancer is 31 to $54 \%$ at age 60 years, with a median age at diagnosis of 37 years (range, 19 to 48 years) in PJS patients [3]. Published European guidelines recommend annual magnetic resonance imaging of the breast from age 25 to 30 years, with mammography being substituted after age 50 years [3], which we endorse.

References

1. Giardiello FM, Brensinger JD, Tersmette AC, et al: Very high risk of cancer in familial Peutz-Jeghers syndrome. Gastroenterology 2000, 119:1447-1453.

2. Hearle N, Schumacher V, Menko FH, et al: Frequency and spectrum of cancers in the Peutz-Jeghers syndrome. Clin Cancer Res 2006, 12:3209-3215.

3. Beggs $A D$, Latchford AR, Vasen HF, et al: Peutz-Jeghers syndrome: a systemic review and recommendations for management. Gut 2010, 59:975-986

P47

PB.47: Mammography in symptomatic women aged 35 to 39 years

WW Chan*, MG Bell, AL Brown

Royal Berkshire NHS Foundation Trust, Reading, UK

Breast Cancer Research 2013, 15(Suppl 1):P47

Introduction: Until recently, in our institution, all women over the age of 35 years referred to the symptomatic breast clinic for triple assessment undergo mammography. Best practice diagnostic guidelines for patients presenting with breast symptoms (Department of Health November 2010) recommend that mammograms should routinely be performed in women aged over 40 years. The aim of this study was to assess the impact that changing our policy would have on breast cancer detection in women aged 35 to 39 years.

Methods: Our Breast Cancer Database identified all women aged 35 to 39 years diagnosed with breast cancer between April 2003 and December 2012. A retrospective review of breast imaging and reports on PACS was carried out to determine whether ultrasound alone would have detected the malignancy.

Results: 106 cancers were diagnosed in 103 women aged 35-39 years. In 103 cases, ultrasound scores using the RCR Breast Group Classification ranged from U3 (indeterminate/probably benign) to U5 (highly suspicious of malignancy). Two patients presented with nipple discharge. One of these patients had mammographic microcalcification due to extensive DCIS with small separate foci of invasive cancer with normal ultrasound examination.

Conclusion: In our institution, one case of extensive DCIS with small separate foci of invasive cancer out of a total of 106 breast cancers would have been missed on breast imaging if routine mammography had not been performed in women aged 35-39 years.

\section{P48}

PB.48: Old habits die hard: breast imaging of symptomatic 35 to 39 year olds

FM Singh", S Gadde

Nightingale Centre and Genesis Prevention Centre, University Hospital of South Manchester NHS Foundation Trust, Wythenshawe, Manchester, UK Breast Cancer Research 2013, 15(Suppl 1):P48

Introduction: The Best Practice Diagnostic Guidelines for Patients Presenting with Breast Symptoms were published in 2010 and suggest patients younger than 40 years should undergo breast ultrasound (US) as first-line imaging with additional mammography for indeterminate and suspicious clinical or US findings. However, our current departmental guidelines are to use firstline mammography in patients aged 35 and over. The aims were to quantify the use of imaging modalities and cancer detection; to assess whether additional information gained by mammography changed management; and to quantify how many fewer mammograms would have been performed if we had adhered to the 2010 Guidelines.

Methods: Imaging and histology reports were reviewed over a 6-month period for 35-year-old to 39-year-old females with a symptomatic breast complaint.

Results: A total of 247 symptomatic 35 to 39 year olds had breast imaging with a malignancy rate of $4 \%$, all cancers presented with a lump/thickening. 
Sixty-nine per cent had both US and mammography, 23\% mammography only and $9 \%$ US only. Four U3 lesions were detected on US with normal mammograms, one of which was malignant. Mammography altered management in three patients, requiring biopsies of the asymptomatic breast, none of which were malignant. If we had adhered to the 2010 Guidelines we would have performed 150 fewer mammograms out of the 171 patients who had both US and mammography.

Conclusion: In our study, no malignancy would have been missed and we would have performed 150 fewer mammograms if we had adhered to the 2010 Guidelines and used US as a first-line investigation.

\section{P49}

PB.49: Preoperative axillary staging in breast cancer patients: repeat sampling in cases of suspicious ultrasound or inconclusive cytology L Clarke, P Newton, A Leaver

Queen Elizabeth Hospital, Gateshead, UK

Breast Cancer Research 2013, 15(Suppl 1):P49

Introduction: In our Trust preoperative axillary staging comprises ultrasound with appearances scored LN1 to LN5, and fine needle aspiration (FNA) of LN3 to LN5 nodes. Repeat sampling with FNA or core biopsy (CB) is performed on inconclusive FNA $\left(\mathrm{C}_{1}, \mathrm{C} 3, \mathrm{C} 4\right)$ at the discretion of the radiologist. Repeat sampling is also performed in $\mathrm{LN} 4 / 5 \mathrm{C} 2$ patients. There has been a trend toward the more invasive and expensive $C B$ for repeat axillary sampling in our unit. Here we assess our practice and the diagnostic yield from repeat sampling, to review current departmental guidelines.

Method: All invasive breast cancer patients who underwent surgery in 2012 were identified from multidisciplinary meeting records. Cytology and histology reports from repeat preoperative sampling and surgery were reviewed.

Results: In 2012, 169 female breast cancer patients underwent axillary FNA and axillary surgery. FNA was followed by CB in $26 \%(44 / 169)$ and no FNAs. Of $\mathrm{C} 1 / \mathrm{C} 3 / \mathrm{C} 4 \mathrm{FNAs}, 34 / 39$ had $\mathrm{CB}$. Of these, $10 / 34$ were malignant. CB was negative in 23 , with $20 / 23$ negative nodes and $3 / 23$ positive nodes at surgery. There were 79 C2 FNAs with 10/79 undergoing CB. Nine of 10 cores were negative, of which one was node-positive at surgery. The $1 / 10$ positive core represented micrometastasis on clearance. No complications resulted from axillary FNA or CB.

Conclusion: Repeat biopsy including CB is useful in $\mathrm{C} 1 / \mathrm{C} 3 / \mathrm{C} 4$ cytology, improving triage to the correct axillary surgical procedure. Repeat biopsy of $\mathrm{C} 2$ nodes with LN4/5 ultrasound has not been validated here: ongoing audit and a higher threshold for repeat biopsy in these patients should be considered.

\section{P50}

PB.50: Axillary lymph node ultrasound in breast cancer patients: what minimum threshold for diffuse cortical thickening predicts nodal involvement?

L Clarke*, A Leaver, P Newton

The Queen Elizabeth Hospital, Gateshead, UK

Breast Cancer Research 2013, 15(Suppl 1):P50

Introduction: In our Trust we classify axillary ultrasound findings as LN1 to LN5, performing fine needle aspiration cytology (FNAC) on LN3 to LN5, where LN3 represents diffuse cortical thickening (DCT) of greater than $2 \mathrm{~mm}$. The resulting FNAC triages patients to either sentinel lymph node biopsy or axillary node dissection. The aim is that patients will undergo only one axillary surgical procedure. There is variation in the literature and between breast units in the DCT threshold for performing FNA, and unnecessary FNAs should be avoided. Does the resulting cytology and surgical histology validate our $2 \mathrm{~mm}$ threshold; or can the threshold be safely increased to $2.3 \mathrm{~mm}$ or $3 \mathrm{~mm}$ as used by some centres?

Methods: The MDT records and images for all our invasive breast cancer patients classified axillary LN3 and operated upon in 2012 were reviewed. The positive predictive value (PPV) was calculated for ranges of DCT for a post-test probability of a $\mathrm{C} 5$ result at FNA, and then for the yield of $2+$ positive nodes at surgery.

Results: A total of 112 female patients were LN3 and underwent FNA in 2012. The PPV for a C5 result in DCT in ranges 2.0 to $2.29,2.30$ to 2.99 and $\geq 3.0$ was $9.1 \%(1 / 11), 11.3 \%(6 / 53)$ and $14.6 \%$ (7/48) respectively. The PPV for a yield of $2+$ malignant nodes was $9.1 \%$ (1/11), 9.4\% (5/53) and 4.2\% (2/48) respectively.

Conclusion: These findings have validated our use of the $2 \mathrm{~mm}$ threshold for FNAC. Increasing the threshold would result in a significant number of women requiring a second axillary surgical procedure as current treatment guidelines stand.

P51

PB.51: Axillary lymph node ultrasound and fine needle aspiration in preoperative staging of breast cancer: re-audit

H Kazi $^{i^{*}}$, H Humphreys $^{2}$, L Clarke ${ }^{2}$, A Robinson ${ }^{2}$, A Leaver $^{2}$

${ }^{1}$ South Tyneside Hospital, South Shields, UK; ${ }^{2}$ Queen Elizabeth Hospital, Gateshead, UK

Breast Cancer Research 2013, 15(Suppl 1):P51

Introduction: Axillary ultrasound and fine needle aspiration (FNA) are used to triage breast cancer patients to appropriate axillary surgery. We present a complete audit cycle. Departmental guidelines were changed to include repeat biopsy in cases with suspicious ultrasound/negative FNA and for patients with inconclusive FNA results between audit periods one and two.

Methods: Retrospective analysis of multidisciplinary meeting and pathology records of all breast cancer patients operated upon in our Trust between January and June 2010, and then between January and December 2012, was reviewed. Descriptive statistics were performed.

Results: Initial audit findings included 125 female patients, with overall combined sensitivity and specificity of ultrasound/FNA being $64 \%(21 / 33)$ and $100 \%(92 / 92)$ respectively. Included in the re-audit were 378 female patients, all underwent axillary ultrasound. Of these, 50.3\% (190/378) patients underwent an FNA with $18.4 \%$ (35/190) having repeat FNA or core biopsy for inconclusive results. Of the repeat biopsies, $80 \%$ (28/35) were benign and 20\% (7/35) were malignant. Ultrasound sensitivity was $78.9 \%$ (86/109). Sensitivity of FNA/biopsy was $82.6 \%$ (71/86). Overall combined sensitivity of ultrasound/FNA was $62.2 \%(74 / 119)$. Ultrasound specificity was $62.1 \%(167 / 269)$. Specificity of FNA was $97.1 \%(101 / 104)$. Overall combined specificity of ultrasound/FNA was $98.9 \%(266 / 269)$.

Conclusion: Performing a repeat FNA/biopsy on inconclusive cases proved valuable in that $80 \%$ patients were spared from surgical morbidity. However, overall sensitivity of preoperative axillary staging has not significantly changed between cycles following resampling. Further audit, analysis and guideline review is needed to ensure optimal patient care.

P52

PB.52: Incidence and clinical significance of focal breast uptake at 18-fluorine fluorodeoxyglucose PET-CT in patients without known breast cancer

P Arce-Calisaya*, J Simpson, N Sharma, A Scarsbrook

Saint James's University Hospital, Leeds, UK

Breast Cancer Research 2013, 15(Suppl 1):P52

Introduction: 18-Fluorine fluorodeoxyglucose (FDG) PET-CT is a sensitive technique and hypermetabolic foci unrelated to the primary malignancy are not infrequently encountered. The aim of this study was to evaluate the clinical significance of unexpected focal FDG uptake within the breast in patients undergoing PET-CT for assessment of other malignancies.

Methods: Consecutive adult patients undergoing FDG PET-CT for assessment of a nonbreast primary cancer between February 2009 and October 2012 were retrospectively reviewed. The incidence of focal FDG uptake within the breast was determined. PET parameters including maximum standardised uptake value (SUVmax), metabolic tumour volume, and total lesion glycolysis were recorded for each patient. The presence and patterns of morphologic changes on CT were assessed. Aetiology and clinical significance were confirmed histologically or by imaging and clinical follow-up.

Results: A total 23 of 8,972 patients $(0.25 \%)$ had unexpected focal FDG uptake in the breast. Twenty patients (86\%) underwent biopsy. Malignancy was confirmed in 17 patients, five patients (22\%) had disseminated disease from their primary cancer and 12 patients $(52 \%)$ had an unsuspected synchronous breast cancer. Eight patients $(67 \%)$ with newly diagnosed breast cancer were outside the screening programme ( $\leq 47 \geq 73$ years). Only 
five patients (42\%) had a breast lesion with SUVmax >4. No PET parameters reliably distinguished benign from malignant pathology.

Conclusion: Incidental focal FDG uptake in the breast is rare but requires further evaluation as approximately $50 \%$ of cases may reflect unsuspected breast malignancy.

\section{P53}

PB.53: Are ethnic minorities more likely to develop triple-negative breast cancer? A systematic review

R Limbada ${ }^{1 *}$, A Slater ${ }^{1}$, AK Jain ${ }^{2}$

${ }^{1}$ De Montfort University, Leicester, UK; ${ }^{2}$ The Nightingale Centre and Genesis Prevention Centre, Wythenshawe Hospital, Manchester, UK

Breast Cancer Research 2013, 15(Suppl 1):P53

Introduction: Triple-negative breast cancer (TNBC) accounts for 10 to $15 \%$ of diagnosed breast cancers worldwide. TNBC is negative for oestrogen receptor, progesterone receptor and human epidermal growth factor receptor 2 expression, and is associated with a poor prognosis. Our study aims to systematically review the possible link between various minority ethnic groups and development of TNBC.

Methods: A systematic review of carefully selected studies was carried out with search of relevant articles in Medline, Science Direct and PubMed from 2005 up to 2013 with studies specifically aimed at TNBC. In the UK, receptor data have not been consistently recorded and involve smaller sample sizes. Hence, the relevant articles include ethnic groups from Asia Pacific and USA. Keywords were: triple negative, TNBC, ethnicity, incidence, prevalence. The data from all relevant articles were combined to give a sufficiently large sample size.

Results: Complete receptor data were available for 72,763 cases, which constitute the sample group. On the available ethnicity data these were divided into five groups: Caucasian $(n=50,797)$, Black $(n=4,969)$, Asian $(n=6,909)$, Hispanic $(n=9,898)$ and other $(n=190)$. Overall there were 9,887 TNBC cases in the sample group with Caucasian $(n=5,956)$, Black $(n=1,341)$, Asian $(n=842)$, Hispanic $(n=1,732)$ and other $(n=16)$. Hence, overall $13.59 \%$ of all diagnosed breast cancers were TNBC with $11.73 \%$ in Caucasian, $26.99 \%$ in Black, $12.19 \%$ in Asian, $17.5 \%$ in Hispanic and $8.42 \%$ in other women.

Conclusion: Overall minority ethnic women have higher incidence of TNBC than Caucasian women with resultant poor prognosis. With rising minority ethnic population in the UK the overall number of TNBC will rise. Further research is required into the reasons for this.

Cite abstracts in this supplement using the relevant abstract number, e.g.: Limbada et al.: PB.53: Are ethnic minorities more likely to develop triple-negative breast cancer? A systematic review. Breast Cancer Research 2013, 15(Suppl 1):P53 